\title{
TOPOLOGICAL SENSITIVITY ANALYSIS FOR TIME-DEPENDENT PROBLEMS
}

\author{
Samuel Amstutz ${ }^{1}$, TAKéo Takahashi ${ }^{2}$ And Boris VeXler $^{3}$
}

\begin{abstract}
The topological sensitivity analysis consists in studying the behavior of a given shape functional when the topology of the domain is perturbed, typically by the nucleation of a small hole. This notion forms the basic ingredient of different topology optimization/reconstruction algorithms. From the theoretical viewpoint, the expression of the topological sensitivity is well-established in many situations where the governing p.d.e. system is of elliptic type. This paper focuses on the derivation of such formulas for parabolic and hyperbolic problems. Different kinds of cost functionals are considered.
\end{abstract}

Mathematics Subject Classification. 49Q10, 49Q12, 35K05, 35L05

Received June 26, 2006. Revised December 5, 2006.

Published online November 21, 2007.

\section{INTRODUCTION}

Consider a domain $\Omega \subset \mathbb{R}^{d}, d=2$ or 3 , and the solution $u_{\Omega}$ of a system of partial differential equations defined in $\Omega$. The topological sensitivity analysis aims at studying the asymptotic behavior of some shape functional of interest $j(\Omega)=J_{\Omega}\left(u_{\Omega}\right)$ with respect to an infinitesimal perturbation of the topology of $\Omega$. This concept was introduced in the field of shape optimization by Schumacher et al. $[14,15,24]$ and was for the first time mathematically justified in $[16,25]$. In these papers, the creation of holes inside the domain is considered. Given a point $x_{0} \in \Omega$, a domain $\omega \subset \mathbb{R}^{d}$ containing the origin and a small perforation $\omega_{\varepsilon}=x_{0}+\varepsilon \omega$, an asymptotic expansion for $\varepsilon$ going to zero is obtained in the form:

$$
j\left(\Omega \backslash \overline{\omega_{\varepsilon}}\right)-j(\Omega)=f(\varepsilon) g\left(x_{0}\right)+o(f(\varepsilon)) .
$$

In this expression, the function $\varepsilon \in \mathbb{R}^{+} \mapsto f(\varepsilon) \in \mathbb{R}^{+}$is smooth and goes to zero with $\varepsilon$. The number $g\left(x_{0}\right)$ is commonly called topological gradient, or topological derivative, at the point $x_{0}$. It gives an indication on the sensitivity of the cost functional with respect to the nucleation of a small hole around $x_{0}$. The map $x \mapsto g(x)$ forms the basis of different kinds of topology optimization algorithms. They mainly rely on the following

\footnotetext{
Keywords and phrases. Topological sensitivity, topology optimization, parabolic equations, hyperbolic equations.

${ }^{1}$ Laboratoire d'analyse non-linéaire et géométrie, Faculté des sciences, 33 rue Louis Pasteur, 84000 Avignon, France; samuel.amstutz@univ-avignon.fr

2 Institut Élie Cartan de Nancy, Nancy-Université, CNRS, INRIA, BP 239, 54506 Vandœuvre-lès-Nancy cedex, France; Takeo.Takahashi@iecn.u-nancy.fr

3 Johann Radon Institute for Computational and Applied Mathematics, Austrian Academy of Sciences, Altenbergerstrasse 69, 4040 Linz, Austria; boris.vexler@oeaw.ac.at
} 
principles. For certain problems, the interpretation in one iteration of some special features of this map, such as peaks, can provide a sufficient information (see e.g. $[7,9,10,18]$ ). In an iterative procedure, the topological gradient can serve as a descent direction for removing matter (see e.g. [16,17,22]). It can also be utilized within a level-set-based algorithm (see e.g. $[1,6,11]$ ).

From the theoretical point of view, most efforts for deriving the expansion (0.1) have been so far focused on problems associated with state equations of elliptic type, for which several generalizations of the above notion have been proposed (e.g. creation of a crack [8], exterior topological derivative [20]). To the best of our knowledge, [9] is the only publication where this issue is addressed for a time-dependent problem. But the proof presented there is merely formal. For instance, convergence theorems for integrals of multivariate functions are used without any checking of their applicability. In addition, a restricted class of cost functional is considered. In another context but still related, one should mention the paper [3], which belongs to a series of works dedicated to the reconstruction of inhomogeneities from boundary measurements (see e.g. [2,4] and the references therein). In these works, asymptotic expansions of the state variable $u_{\Omega}$ at the location of the measurements or its integrals against special test functions are derived. Then techniques borrowed from signal processing are used to recover some features of the unknown inclusions. In the frame of topology optimization, one would like to be able to deal with general cost functionals, which makes the analysis quite different. In particular, an adjoint method is generally appreciated for computational convenience.

The present paper investigates the topological sensitivity analysis of shape functionals for governing PDEs of parabolic and hyperbolic types. For simplicity, the mathematical developments are presented for model problems. The following heat and wave equations for an inclusion are considered:

$$
\rho_{\varepsilon} \frac{\partial^{p} u_{\varepsilon}}{\partial t^{p}}-\operatorname{div}\left(\alpha_{\varepsilon} A \nabla u_{\varepsilon}\right)=F_{\varepsilon}, \quad p=1,2
$$

The coefficients $\rho_{\varepsilon}$ and $\alpha_{\varepsilon}$ are positive and piecewise constant, with values inside the inclusion $\omega_{\varepsilon}$ different from those of the background medium. The right hand side $F_{\varepsilon}$ should be smooth in $\omega_{\varepsilon}$ and its complementary, $A$ denotes some symmetric positive definite matrix. Dirichlet boundary conditions on the external border of $\Omega$ and null initial conditions are prescribed. For these problems, a large class of cost functionals is treated. The calculus of their sensitivity is performed by means of an adjoint state method, which, in addition to the practical interest, enables to write the expansion (0.1) in a unified form. This setting allows for some straightforward generalizations. First, the same results hold for other kinds of linear boundary conditions on $\partial \Omega$ (e.g. of Neumann or Robin type), since they play no role in the analysis except that of guaranteeing well-posedness and regularity properties. Second, the formulas corresponding to a vector-valued state variable can be easily inferred, provided that the expression of the first order polarization tensor (also called Pólya-Szegö polarization tensor, or virtual mass) is known. This notion is however well-documented (see e.g. [2,4]). Third, the case where $\omega_{\varepsilon}$ is a hole with Neumann boundary condition can be obtained by taking in the final formulas $\rho_{\varepsilon}$ and $\alpha_{\varepsilon}$ to be zero inside $\omega_{\varepsilon}$ and the associated polarization tensor. This statement is proved in [5] for elliptic problems. Here, the proof, which is very similar, is omitted. We also point out that the interest of our result has already been illustrated by promising numerical experiments $[7,9]$. Those concern nondestructive testing in elastic media with acoustic waves and a least-square-type cost function. In [7], the expression of the topological gradient in the time domain was formally deduced from the harmonic case through the Fourier transform. This formula, identical to that found in [9], is retrieved as a particular case.

The rest of this article is organized as follows. In Section 1, we recall an abstract result which provides in a general setting the structure of the topological asymptotic expansion. In Sections 2, 3 and 4, we present our main result for the heat equation. Some examples of cost functionals are exhibited in Section 5 . Sections 6 through 12 contain the proofs. Sections 13 through 17 are devoted to the wave equation, following the same outline. 


\section{A preliminary RESUlt}

Let $X$ and $X_{0} \subset X$ be two Banach spaces. For all parameter $\varepsilon \in\left[0, \varepsilon_{0}\right), \varepsilon_{0}>0$, we consider a function $u_{\varepsilon} \in X_{0}$ solving a variational problem of the form

$$
\mathcal{A}_{\varepsilon}\left(u_{\varepsilon}, v\right)=\mathcal{L}_{\varepsilon}(v) \quad \forall v \in X
$$

where $\mathcal{A}_{\varepsilon}: X \times X \rightarrow \mathbb{R}$, and $\mathcal{L}_{\varepsilon}: X \rightarrow \mathbb{R}$ are a bilinear form on $X$ and a linear functional on $X$, respectively. We also consider a functional $\mathcal{J}_{\varepsilon}: X_{0} \rightarrow \mathbb{R}$ and the associated reduced cost functional

$$
j(\varepsilon)=\mathcal{J}_{\varepsilon}\left(u_{\varepsilon}\right) \in \mathbb{R} .
$$

Suppose also that there exists a function $f: \mathbb{R} \rightarrow \mathbb{R}$ such that

$$
\lim _{\varepsilon \rightarrow 0} f(\varepsilon)=0
$$

and such that the following holds.

(1) There exist $D \mathcal{J}_{\varepsilon}\left(u_{0}\right) \in X_{0}^{\prime}$ and $\delta \mathcal{J} \in \mathbb{R}$ such that

$$
\mathcal{J}_{\varepsilon}\left(u_{\varepsilon}\right)=\mathcal{J}_{0}\left(u_{0}\right)+\left\langle D \mathcal{J}_{\varepsilon}\left(u_{0}\right), u_{\varepsilon}-u_{0}\right\rangle_{X_{0}^{\prime}, X_{0}}+f(\varepsilon) \delta \mathcal{J}+o(f(\varepsilon)),
$$

when $\varepsilon$ goes to zero. Here $X_{0}^{\prime}$ denotes the dual space of $X_{0}$ and $\langle., .\rangle_{X_{0}^{\prime}, X_{0}}$ is the corresponding duality pairing. The notation $D \mathcal{J}_{\varepsilon}\left(u_{0}\right)$ has been used for the reader's convenience since in most applications, it coincides with the Fréchet derivative of $\mathcal{J}_{\varepsilon}$ evaluated at $u_{0}$.

(2) There exists $v_{\varepsilon} \in X$ solving the adjoint equation

$$
\mathcal{A}_{\varepsilon}\left(\varphi, v_{\varepsilon}\right)=-\left\langle D \mathcal{J}_{\varepsilon}\left(u_{0}\right), \varphi\right\rangle_{X_{0}^{\prime}, X_{0}} \quad \forall \varphi \in X_{0} .
$$

(3) There exist $\delta \mathcal{A}, \delta \mathcal{L} \in \mathbb{R}$ such that for $\varepsilon$ going to zero,

$$
\begin{gathered}
\left(\mathcal{A}_{\varepsilon}-\mathcal{A}_{0}\right)\left(u_{0}, v_{\varepsilon}\right)=f(\varepsilon) \delta \mathcal{A}+o(f(\varepsilon)), \\
\left(\mathcal{L}_{\varepsilon}-\mathcal{L}_{0}\right)\left(v_{\varepsilon}\right)=f(\varepsilon) \delta \mathcal{L}+o(f(\varepsilon)) .
\end{gathered}
$$

Proposition 1.1. Under the above assumptions, we have the following asymptotic expansion for $\varepsilon$ tending to zero:

$$
j(\varepsilon)-j(0)=f(\varepsilon)(\delta \mathcal{A}-\delta \mathcal{L}+\delta \mathcal{J})+o(f(\varepsilon)) .
$$

For the proof, see [5].

\section{Part 1. Topological sensitivity analysis for parabolic problems}

\section{Setting of the PROBlem}

Let $\Omega$ be a bounded domain of $\mathbb{R}^{d}, d=2$ or 3 , with smooth $\left(C^{\infty}\right)$ boundary $\partial \Omega$. We consider a small subdomain $\omega_{\varepsilon}=x_{0}+\varepsilon \omega$, where $x_{0} \in \Omega$ and $\omega \subset \mathbb{R}^{d}$ is a bounded domain containing the origin with smooth and connected boundary $\partial \omega$.

Let $A$ be a symmetric positive definite matrix and let $\alpha_{0}, \alpha_{1}, \rho_{0}, \rho_{1}$ be some positive real numbers. For every parameter $\varepsilon \in\left[0, \varepsilon_{0}\right), \varepsilon_{0}$ small enough, we define the piecewise constant coefficients

$$
\alpha_{\varepsilon}=\left\{\begin{array}{ll}
\alpha_{1} & \text { in } \omega_{\varepsilon} \\
\alpha_{0} & \text { in } \Omega \backslash \overline{\omega_{\varepsilon}}
\end{array}, \quad \rho_{\varepsilon}=\left\{\begin{array}{ll}
\rho_{1} & \text { in } \omega_{\varepsilon} \\
\rho_{0} & \text { in } \Omega \backslash \overline{\omega_{\varepsilon}}
\end{array} .\right.\right.
$$


Given $F_{0}, F_{1} \in L^{2}\left(0, T ; H^{-1}(\Omega)\right)$, we also define the function

$$
F_{\varepsilon}= \begin{cases}F_{1} & \text { in } \omega_{\varepsilon} \times(0, T) \\ F_{0} & \text { in }\left(\Omega \backslash \overline{\omega_{\varepsilon}}\right) \times(0, T) .\end{cases}
$$

We consider the following heat equation:

$$
\left\{\begin{aligned}
\rho_{\varepsilon} \frac{\partial u_{\varepsilon}}{\partial t}-\operatorname{div}\left(\alpha_{\varepsilon} A \nabla u_{\varepsilon}\right) & =F_{\varepsilon} & & \text { in } \Omega \times(0, T), \\
u_{\varepsilon} & =0 & & \text { on } \partial \Omega \times(0, T), \\
u_{\varepsilon}(\cdot, 0) & =0 & & \text { in } \Omega .
\end{aligned}\right.
$$

The corresponding variational formulation for

$$
\begin{gathered}
X=L^{2}\left(0, T ; H_{0}^{1}(\Omega)\right) \cap H^{1}\left(0, T ; H^{-1}(\Omega)\right), \\
u_{\varepsilon} \in X_{0}=\{u \in X, u(., 0)=0\}
\end{gathered}
$$

can be written as:

$$
\int_{0}^{T}\left\langle\rho_{\varepsilon} \frac{\partial u_{\varepsilon}}{\partial t}, v\right\rangle_{H^{-1}(\Omega), H_{0}^{1}(\Omega)} \mathrm{d} t+\int_{0}^{T} a_{\varepsilon}\left(u_{\varepsilon}, v\right) \mathrm{d} t=\int_{0}^{T} \ell_{\varepsilon}(v) \mathrm{d} t \quad \forall v \in X .
$$

Here, the bilinear form $a_{\varepsilon}$ and the linear functional $\ell_{\varepsilon}$ are defined by:

$$
\begin{gathered}
a_{\varepsilon}(u, v)=\int_{\Omega} \alpha_{\varepsilon} A \nabla u \cdot \nabla v \mathrm{~d} x, \\
\ell_{\varepsilon}(v)=\int_{\Omega} F_{\varepsilon} v \mathrm{~d} x .
\end{gathered}
$$

Equation (2.2) can be identified with the generic form (1.1) by setting

$$
\begin{gathered}
\mathcal{A}_{\varepsilon}(u, v)=\int_{0}^{T}\left(\left\langle\rho_{\varepsilon} \frac{\partial u}{\partial t}, v\right\rangle_{H^{-1}(\Omega), H_{0}^{1}(\Omega)}+a_{\varepsilon}(u, v)\right) \mathrm{d} t, \\
\mathcal{L}_{\varepsilon}(v)=\int_{0}^{T} \ell_{\varepsilon}(v) \mathrm{d} t .
\end{gathered}
$$

To apply the result of Section 1, we deal with a cost function of the form

$$
j(\varepsilon)=\mathcal{J}_{\mathcal{\varepsilon}}\left(u_{\varepsilon}\right)=\int_{0}^{T} J_{\varepsilon}\left(u_{\varepsilon}\right) \mathrm{d} t
$$

where the functional $J_{\varepsilon}: H_{0}^{1}(\Omega) \rightarrow \mathbb{R}$ satisfies the following assumptions:

$$
\begin{gathered}
J_{\varepsilon}(u) \in L^{1}(0, T) \quad \forall u \in X, \forall \varepsilon \in\left[0, \varepsilon_{0}\right), \\
\mathcal{J}_{\varepsilon}\left(u_{\varepsilon}\right)=\mathcal{J}_{\varepsilon}\left(u_{0}\right)+\int_{0}^{T}\left\langle D J_{\varepsilon}\left(u_{0}\right), u_{\varepsilon}-u_{0}\right\rangle_{H^{-1}(\Omega), H_{0}^{1}(\Omega)} \mathrm{d} t+\varepsilon^{d} \delta \mathcal{J}_{1}+o\left(\varepsilon^{d}\right), \\
\mathcal{J}_{\varepsilon}\left(u_{0}\right)=\mathcal{J}_{0}\left(u_{0}\right)+\varepsilon^{d} \delta \mathcal{J}_{2}+o\left(\varepsilon^{d}\right), \\
\left\|D J_{\varepsilon}\left(u_{0}\right)-D J_{0}\left(u_{0}\right)\right\|_{L^{2}\left(0, T ; H^{-1}(\Omega)\right)}=o\left(\varepsilon^{d / 2}\right),
\end{gathered}
$$

with $D J_{\varepsilon}\left(u_{0}(t)\right) \in H^{-1}(\Omega)$ for almost all $t \in(0, T)$. These assumptions will be checked for some typical cost functionals in Section 5 . 


\section{Remarks 2.1.}

(1) Like in Section 1, we use the notation $D J_{\varepsilon}\left(u_{0}(\cdot, t)\right)$ since in most applications, it coincides with the Fréchet derivative of $J_{\varepsilon}$ evaluated at $u_{0}(\cdot, t)$.

(2) For simplicity, we do not consider the case where the cost functional $J_{\varepsilon}$ depends explicitly on time. However, all the analysis could be easily adapted to this case.

We introduce the adjoint state $v_{\varepsilon} \in X$ defined by (1.4), i.e.,

$$
\int_{0}^{T}\left\langle\rho_{\varepsilon} \frac{\partial \varphi}{\partial t}, v_{\varepsilon}\right\rangle_{H^{-1}(\Omega), H_{0}^{1}(\Omega)} \mathrm{d} t+\int_{0}^{T} a_{\varepsilon}\left(\varphi, v_{\varepsilon}\right) \mathrm{d} t=-\int_{0}^{T} D J_{\varepsilon}\left(u_{0}\right) \varphi \mathrm{d} t \quad \forall \varphi \in X_{0} .
$$

The strong formulation of the PDE associated with (2.10) reads

$$
\left\{\begin{aligned}
-\rho_{\varepsilon} \frac{\partial v_{\varepsilon}}{\partial t}-\operatorname{div}\left(\alpha_{\varepsilon} A \nabla v_{\varepsilon}\right) & =-D J_{\varepsilon}\left(u_{0}\right) & & \text { in } \Omega \times(0, T), \\
v_{\varepsilon} & =0 & & \text { on } \partial \Omega \times(0, T), \\
v_{\varepsilon}(\cdot, T) & =0 & & \text { in } \Omega .
\end{aligned}\right.
$$

\section{Regularity ASSUMPtions}

To enable the analysis, we make additional regularity assumptions, namely: there exist two neighborhoods $\Omega_{F}$ and $\Omega_{J}$ of $x_{0}$ such that

$$
\begin{gathered}
F_{0} \in L^{2}\left(0, T ; H^{4}\left(\Omega_{F}\right)\right) \cap H^{2}\left(0, T ; L^{2}\left(\Omega_{F}\right)\right), \\
F_{1} \in L^{2}\left(0, T ; W^{1, \infty}\left(\Omega_{F}\right)\right), \\
D J_{0}\left(u_{0}\right) \in L^{2}\left(0, T ; H^{4}\left(\Omega_{J}\right)\right) \cap H^{2}\left(0, T ; L^{2}\left(\Omega_{J}\right)\right) .
\end{gathered}
$$

The condition (3.3) will be checked for the examples of cost functional presented in Section 5. The conditions (3.1) and (3.2) are assumed throughout all this part of the paper. Then we get the following regularity on the direct and adjoint solutions. The proof is given in Section 6 .

Proposition 3.1. Assume that $u_{0}$ and $v_{0}$ solve (2.1) and (2.11), respectively, for $\varepsilon=0$ and that the regularity assumptions (3.1), (3.3) hold. Then for all subdomains $\widetilde{\Omega}_{F} \subset \subset \Omega_{F}, \widetilde{\Omega}_{J} \subset \subset \Omega_{J}$, we have

$$
\begin{aligned}
& u_{0} \in L^{2}\left(0, T, H^{6}\left(\widetilde{\Omega}_{F}\right)\right) \cap H^{3}\left(0, T ; L^{2}\left(\widetilde{\Omega}_{F}\right)\right), \\
& v_{0} \in L^{2}\left(0, T, H^{6}\left(\widetilde{\Omega}_{J}\right)\right) \cap H^{3}\left(0, T ; L^{2}\left(\widetilde{\Omega}_{J}\right)\right) .
\end{aligned}
$$

For the sake of readability, we fix some subdomain $\widetilde{\Omega}$ containing $x_{0}$ and such that $\widetilde{\Omega} \subset \subset \Omega_{F}, \widetilde{\Omega} \subset \subset \Omega_{J}$, and we remember in the sequel that

$$
\begin{gathered}
F_{0}, F_{1} \in L^{2}\left(0, T ; W^{1, \infty}(\widetilde{\Omega})\right), \\
u_{0}, v_{0} \in L^{2}\left(0, T, H^{6}(\widetilde{\Omega})\right) \cap H^{3}\left(0, T ; L^{2}(\widetilde{\Omega})\right) .
\end{gathered}
$$

In particular, by interpolation (see [19], Chap. 4, Prop. 2.3), it follows

$$
u_{0}, v_{0} \in H^{1}\left(0, T, H^{4}(\widetilde{\Omega})\right)
$$

The domains $\Omega_{F}, \Omega_{J}, \widetilde{\Omega}_{F}$ and $\widetilde{\Omega}_{J}$ will only be distinguished when studying special cost functionals. 


\section{Main Result}

In order to state the main result, we first introduce the polarization matrix $\mathcal{P}_{\omega, r} \in \mathbb{R}^{d \times d}, r \in \mathbb{R}^{+}$. It is defined as follows:

(1) if $r=1$, then $\mathcal{P}_{\omega, 1}=0$;

(2) otherwise, it has the entries

$$
\left(\mathcal{P}_{\omega, r}\right)_{i j}=\int_{\partial \omega} p_{j} x_{i} \mathrm{~d} s
$$

where $x_{j}$ is the $j^{\text {th }}$ coordinate of the point $x$ and the density $p_{i}$ associated with the $i^{\text {th }}$ basis vector $e_{i}$ of $\mathbb{R}^{d}$ is the unique solution of the boundary integral equation

$$
\frac{r+1}{r-1} \frac{p_{i}(x)}{2}+\int_{\partial \omega} p_{i}(y) A \nabla E(x-y) \cdot n(x) \mathrm{d} s(y)=A e_{i} \cdot n(x) \quad \forall x \in \partial \omega .
$$

Here, $E$ denotes the fundamental solution of the operator $u \mapsto-\operatorname{div}(A \nabla u)$. We recall that the matrix $\mathcal{P}_{\omega, r}$ is symmetric (see, e.g., [4]).

To apply the abstract result of Section 1, we first provide the following lemmas, which will be proved in Sections 7 through 11.

Lemma 4.1. Assume that the bilinear form $a_{\varepsilon}$ is defined by (2.3), that $u_{0}$ and $v_{\varepsilon}$ solve (2.1) and (2.11), respectively, that we have the regularity assumptions (3.1)-(3.3) and that (2.9) holds true. Then

$$
\int_{0}^{T}\left(a_{\varepsilon}-a_{0}\right)\left(u_{0}, v_{\varepsilon}\right) \mathrm{d} t=\varepsilon^{d} \delta a+o\left(\varepsilon^{d}\right),
$$

with

$$
\delta a=\alpha_{0} \int_{0}^{T} \nabla u_{0}\left(x_{0}, t\right) \cdot \mathcal{P}_{\omega, \frac{\alpha_{1}}{\alpha_{0}}} \nabla v_{0}\left(x_{0}, t\right) \mathrm{d} t .
$$

Lemma 4.2. Assume that $u_{0}$ and $v_{\varepsilon}$ solve (2.1) and (2.11), respectively, that we have the regularity assumptions (3.1)-(3.3) and that (2.9) holds true. Then

$$
\int_{0}^{T}\left\langle\left(\rho_{\varepsilon}-\rho_{0}\right) \frac{\partial u_{0}}{\partial t}, v_{\varepsilon}\right\rangle_{H^{-1}(\Omega), H_{0}^{1}(\Omega)} \mathrm{d} t=\varepsilon^{d} \delta \rho+o\left(\varepsilon^{d}\right),
$$

with

$$
\delta \rho=\left(\rho_{1}-\rho_{0}\right)|\omega| \int_{0}^{T} \frac{\partial u_{0}}{\partial t}\left(x_{0}, t\right) v_{0}\left(x_{0}, t\right) \mathrm{d} t .
$$

Lemma 4.3. Assume that the linear functional $\ell_{\varepsilon}$ is defined by (2.4) and that $u_{0}$ and $v_{\varepsilon}$ solve (2.1) and (2.11), respectively, that we have the regularity assumptions (3.1)-(3.3) and that (2.9) holds true. Then

$$
\int_{0}^{T}\left(\ell_{\varepsilon}-\ell_{0}\right)\left(v_{\varepsilon}\right) \mathrm{d} t=\varepsilon^{d} \delta \ell+o\left(\varepsilon^{d}\right)
$$

with

$$
\delta \ell=|\omega| \int_{0}^{T}\left(F_{1}\left(x_{0}, t\right)-F_{0}\left(x_{0}, t\right)\right) v_{0}\left(x_{0}, t\right) \mathrm{d} t .
$$

We are now in position to state the main result of this part. 
Theorem 4.1. Assume that the cost functional $\mathcal{J}$ satisfies (2.5)-(2.9). Suppose moreover that $u_{0}$ and $v_{0}$ solve (2.1) and (2.11), respectively, for $\varepsilon=0$ and that the regularity assumptions (3.1)-(3.3) hold. Then we have the following asymptotic expansion:

$$
\begin{array}{r}
j(\varepsilon)-j(0)=\varepsilon^{d}\left[\left(\rho_{1}-\rho_{0}\right)|\omega| \int_{0}^{T} \frac{\partial u_{0}}{\partial t}\left(x_{0}, t\right) v_{0}\left(x_{0}, t\right) \mathrm{d} t+\alpha_{0} \int_{0}^{T} \nabla u_{0}\left(x_{0}, t\right) \cdot \mathcal{P}_{\omega, \frac{\alpha_{1}}{\alpha_{0}}} \nabla v_{0}\left(x_{0}, t\right) \mathrm{d} t\right. \\
\left.-|\omega| \int_{0}^{T}\left(F_{1}\left(x_{0}, t\right)-F_{0}\left(x_{0}, t\right)\right) v_{0}\left(x_{0}, t\right) \mathrm{d} t+\delta \mathcal{J}_{1}+\delta \mathcal{J}_{2}\right]+o\left(\varepsilon^{d}\right) .
\end{array}
$$

This theorem is a direct consequence of Proposition 1.1 combined with the above lemmas and the definitions $\delta \mathcal{A}=\delta \rho+\delta a, \delta \mathcal{L}=\delta \ell, \delta \mathcal{J}=\delta \mathcal{J}_{1}+\delta \mathcal{J}_{2}$

$$
\left\langle D \mathcal{J}_{\varepsilon}\left(u_{0}\right), \varphi\right\rangle_{X_{0}^{\prime}, X_{0}}=\int_{0}^{T}\left\langle D J_{\varepsilon}\left(u_{0}(\cdot, t)\right), \varphi(t)\right\rangle_{H^{-1}(\Omega), H_{0}^{1}(\Omega)} \mathrm{d} t .
$$

\section{Remarks 4.1.}

(1) The polarization matrix can be determined analytically in some cases. For instance, we have for the Laplace operator ( $A$ is the identity matrix) and $\omega=B(0,1)$ :

$$
\begin{gathered}
\mathcal{P}_{\omega, r}=2|\omega| \frac{r-1}{r+1} \mathrm{I}_{2} \quad \text { in 2D (disc), } \\
\mathcal{P}_{\omega, r}=3|\omega| \frac{r-1}{r+2} \mathrm{I}_{3} \quad \text { in 3D (sphere), }
\end{gathered}
$$

where $I_{2}, I_{3}$ denote the identity matrices in dimensions 2 and 3, respectively. For more details on polarization matrices see, e.g., $[2,4,5,21,23]$ and the references therein.

(2) Theorem 4.1 can be extended to some other situations. First, on the external boundary $\partial \Omega$, we can replace the Dirichlet condition by any kind of linear boundary condition guaranteeing well-posedness of the direct and adjoint PDEs, like the Neumann or the Robin boundary condition. Second, the proof can be easily adapted to other parabolic equations or systems like for instance the Stokes system.

(3) Theorem 4.1 remains valid in the case of a hole with Neumann condition on its boundary. The corresponding topological asymptotic expansion is given by (4.6) with $\rho_{1}=0, \alpha_{1}=0, F_{1}=0$ and the polarization matrix computed by solving (4.2) for $r=0$ (see, e.g., [4,5] for more details).

In the next section we present some examples of cost functional $\mathcal{J}$ satisfying the assumptions of the theorem.

\section{Examples of COST FUnCtional}

The proofs of the following results are given in Section 12.

Theorem 5.1. Assume that $J_{\varepsilon} \in \mathcal{C}^{2}\left(L^{2}(\Omega), \mathbb{R}\right)$ (in the sense of Fréchet) and satisfies, for all $M \geq 0$,

$$
\sup _{\|v\|_{L^{2}(\Omega)} \leq M}\left\|D^{2} J_{\varepsilon}(v)\right\|_{\mathcal{B}\left(L^{2}(\Omega)\right)} \leq C(M)
$$

with a positive constant $C(M)$ which does not depend on $\varepsilon$ and with $\mathcal{B}\left(L^{2}(\Omega)\right)$ denoting the space of bilinear forms on $L^{2}(\Omega)$.

Then $\mathcal{J}_{\varepsilon}$ is well-defined on $X$ and fulfills $(2.7)$ with $\delta \mathcal{J}_{1}=0$. 
Corollary 5.1. The asymptotic expansion (4.6) holds true for the following cost functionals with the values of $\delta \mathcal{J}_{1}$ and $\delta \mathcal{J}_{2}$ given below.

(1) For the functional

$$
J_{\varepsilon}(u)=\int_{\Omega}\left|u-u_{d}\right|^{2} \mathrm{~d} x
$$

with $u_{d} \in L^{2}(\Omega) \cap H^{4}\left(B\left(x_{0}, R\right)\right), R>0$, we have $\delta \mathcal{J}_{1}=0$ and $\delta \mathcal{J}_{2}=0$. Note that the creation of a hole with Neumann condition on its boundary cannot be considered for this functional, cf. Remark 4.1 (3).

(2) For the functional

$$
J_{\varepsilon}(u)=\int_{\Omega} \alpha_{\varepsilon}\left|u-u_{d}\right|^{2} \mathrm{~d} x
$$

with $u_{d} \in L^{2}(\Omega) \cap H^{4}\left(B\left(x_{0}, R\right)\right), R>0$, we have $\delta \mathcal{J}_{1}=0$ and

$$
\delta \mathcal{J}_{2}=\left(\alpha_{1}-\alpha_{0}\right)|\omega| \int_{0}^{T}\left|u_{0}\left(x_{0}, t\right)-u_{d}\left(x_{0}\right)\right|^{2} \mathrm{~d} t .
$$

We end this section by giving two other examples of cost functional which are not included in the setting of Theorem 5.1.

Proposition 5.1. The asymptotic expansion (4.6) holds true for the following cost functionals.

(1) For the functional

$$
J_{\varepsilon}(u)=\int_{\Omega} \eta(x) A \nabla\left(u-u_{d}\right) \cdot \nabla\left(u-u_{d}\right) \mathrm{d} x
$$

where $u_{d} \in L^{2}\left(0, T ; H^{1}(\Omega)\right)$ and $\eta$ is a smooth $\left(\mathcal{C}^{\infty}\right)$ function whose support does not contain $x_{0}$, we have $\delta \mathcal{J}_{1}=0$ and $\delta \mathcal{J}_{2}=0$.

(2) If we replace in (2.1) the Dirichlet boundary condition on $\partial \Omega$ by the Neumann boundary condition (for instance), then it makes sense to consider the functional

$$
\mathcal{J}_{\varepsilon}(u)=\int_{0}^{T} \int_{\partial \Omega}\left|u-u_{d}\right|^{2} \mathrm{~d} s \mathrm{~d} t
$$

where $u_{d} \in L^{2}\left(0, T ; L^{2}(\partial \Omega)\right)$. We have $\delta \mathcal{J}_{1}=0$ and $\delta \mathcal{J}_{2}=0$.

The subsequent sections are devoted to the proofs of the results previously stated.

\section{Regularity RESUlts}

Proposition 3.1 is a straightforward application of the following lemma.

Lemma 6.1. Let $\widetilde{\Omega} \subset \subset \Omega, k$ be a positive integer, $f \in L^{2}\left(0, T ; H^{-1}(\Omega)\right) \cap L^{2}\left(0, T ; H^{k}(\widetilde{\Omega})\right) \cap H^{k / 2}\left(0, T ; L^{2}(\widetilde{\Omega})\right)$, $g \in L^{2}\left(0, T ; H^{1 / 2}(\partial \Omega)\right)$ and $z$ be the solution of the system:

$$
\left\{\begin{aligned}
\rho_{0} \frac{\partial z}{\partial t}-\operatorname{div}\left(\alpha_{0} A \nabla z\right) & =f & & \text { in } \Omega \times(0, T), \\
z & =g & & \text { on } \partial \Omega \times(0, T), \\
z(\cdot, 0) & =0 & & \text { in } \Omega .
\end{aligned}\right.
$$

Then, for all subdomain $\Omega_{k} \subset \subset \widetilde{\Omega}$, we have

$$
z \in L^{2}\left(0, T ; H^{k+2}\left(\Omega_{k}\right)\right) \cap H^{k / 2+1}\left(0, T ; L^{2}\left(\Omega_{k}\right)\right) .
$$

The same result holds if the Dirichlet boundary condition on $\partial \Omega$ is replaced by a Neumann or Robin condition of the form $\frac{\partial z}{\partial n}+\lambda z=g, \lambda \in \mathbb{R}, g \in L^{2}\left(0, T ; H^{-1 / 2}(\partial \Omega)\right)$. 
Proof. The difficulty comes from the fact that the so-called compatibility relations required to apply the standard parabolic regularity theorems are not satisfied here. We will construct auxiliary functions for which those relations hold. Our proof follows a bootstrapping argument.

(1) We introduce a domain $\Omega_{0}$ such that $\Omega_{k} \subset \subset \Omega_{0} \subset \subset \widetilde{\Omega}$. Let $\eta_{0}$ be a smooth function with

$$
\begin{aligned}
& \eta_{0}=0 \text { in } \Omega \backslash \overline{\widetilde{\Omega}} \\
& \eta_{0}=1 \text { in } \Omega_{0} .
\end{aligned}
$$

We consider the function

$$
z_{0}=\eta_{0} z
$$

It solves:

$$
\left\{\begin{aligned}
\rho_{0} \frac{\partial z_{0}}{\partial t}-\operatorname{div}\left(\alpha_{0} A \nabla z_{0}\right) & =f_{0} & & \text { in } \Omega \times(0, T), \\
z_{0} & =0 & & \text { on } \partial \Omega \times(0, T), \\
z_{0}(\cdot, 0) & =0 & & \text { in } \Omega,
\end{aligned}\right.
$$

with

$$
f_{0}=\eta_{0} f-2 \alpha_{0} A \nabla \eta_{0} . \nabla z-\eta_{0} \operatorname{div}\left(\alpha_{0} A \nabla \eta_{0}\right) z .
$$

We are guaranteed the minimal regularity $z \in L^{2}\left(0, T ; H^{1}(\Omega)\right)$, from which we deduce that $f_{0} \in$ $L^{2}\left(0, T ; L^{2}(\Omega)\right)$. Using [19], Chapter 4, Theorem 1.1, we derive that $z_{0} \in L^{2}\left(0 ; T, H^{2}(\Omega)\right) \cap H^{1}\left(0, T ; L^{2}(\Omega)\right)$, and consequently that

$$
z \in L^{2}\left(0, T ; H^{2}\left(\Omega_{0}\right)\right) \cap H^{1}\left(0, T ; L^{2}\left(\Omega_{0}\right)\right) .
$$

(2) Assume that, given an integer $p \in\{0, \ldots, k-1\}$, there exists a domain $\Omega_{p}$, with $\Omega_{k} \subset \subset \Omega_{p} \subset \subset \widetilde{\Omega}$, such that

$$
z \in L^{2}\left(0, T ; H^{p+2}\left(\Omega_{p}\right)\right) \cap H^{p / 2+1}\left(0, T ; L^{2}\left(\Omega_{p}\right)\right) .
$$

If $p+1<k$, we define a domain $\Omega_{p+1}$ such that $\Omega_{k} \subset \subset \Omega_{p+1} \subset \subset \Omega_{p}$. We introduce a smooth function $\eta_{p+1}$ satisfying

and we define the function

$$
\begin{aligned}
& \eta_{p+1}=0 \text { in } \Omega \backslash \overline{\Omega_{p}} \\
& \eta_{p+1}=1 \text { in } \Omega_{p+1},
\end{aligned}
$$

$$
z_{p+1}=\eta_{p+1} z
$$

It solves

$$
\left\{\begin{aligned}
\rho_{0} \frac{\partial z_{p+1}}{\partial t}-\operatorname{div}\left(\alpha_{0} A \nabla z_{p+1}\right) & =f_{p+1} & & \text { in } \Omega \times(0, T), \\
z_{p+1} & =0 & & \text { on } \partial \Omega \times(0, T), \\
z_{p+1}(\cdot, 0) & =0 & & \text { in } \Omega
\end{aligned}\right.
$$

with

$$
f_{p+1}=\eta_{p+1} f-2 \alpha_{0} A \nabla \eta_{p+1} . \nabla z-\eta_{p+1} \operatorname{div}\left(\alpha_{0} A \nabla \eta_{p+1}\right) z .
$$

Using [19], Chapter 4, Proposition 2.3, we obtain that $f_{p+1} \in L^{2}\left(0, T ; H^{p+1}\left(\Omega_{p}\right)\right) \cap H^{(p+1) / 2}\left(0, T ; L^{2}\left(\Omega_{p}\right)\right)$. It follows (see [19], Chap. 4 , Th. 5.3) that $z_{p+1} \in L^{2}\left(0, T ; H^{p+3}\left(\Omega_{p}\right)\right) \cap H^{(p+3) / 2}\left(0, T ; L^{2}\left(\Omega_{p}\right)\right)$, and thus that

$$
z \in L^{2}\left(0, T ; H^{p+3}\left(\Omega_{p+1}\right)\right) \cap H^{(p+3) / 2}\left(0, T ; L^{2}\left(\Omega_{p+1}\right)\right) .
$$

Hence the relation (6.5) holds true at rank $p+1$. The relation (6.2) is obtained by repeating this procedure up to the rank $p+1=k$. 


\section{Auxiliary Results on elliptic problems}

We start by introducing a vector field $H=\left(H_{1}, \ldots, H_{d}\right)^{\top}$ where the components $H_{i}$ are given as the solutions of the system:

$$
\left\{\begin{array}{rlll}
\operatorname{div}\left(A \nabla H_{i}\right) & =0 & & \text { in } \omega, \\
\operatorname{div}\left(A \nabla H_{i}\right) & =0 & & \text { in } \mathbb{R}^{d} \backslash \bar{\omega}, \\
H_{i}^{+}-H_{i}^{-} & =0 & & \text { on } \partial \omega, \\
\alpha_{1}\left(A \nabla H_{i} \cdot n\right)^{+}-\alpha_{0}\left(A \nabla H_{i} \cdot n\right)^{-} & =\left(\alpha_{1}-\alpha_{0}\right)(A n)_{i} & & \text { on } \partial \omega, \\
H_{i} & \rightarrow 0 & \text { at } \infty .
\end{array}\right.
$$

In the above equations, $n=\left(n_{1}, \ldots, n_{d}\right)^{\top}$ denotes the outer unit normal of $\omega$ and the superscripts + and indicate the traces of the restriction to $\omega$ and to $\mathbb{R}^{d} \backslash \bar{\omega}$, respectively.

The solution $H_{i}$ can be expressed by means of a single layer potential (see, e.g., [4,13]), namely, there exists $p_{i} \in H^{-1 / 2}(\partial \omega)$ such that

$$
\begin{gathered}
\int_{\partial \omega} p_{i} \mathrm{~d} s(y)=0, \\
H_{i}(x)=\int_{\partial \omega} p_{i}(y) E(x-y) \mathrm{d} s(y),
\end{gathered}
$$

for all $x \in \mathbb{R}^{d}$. To determine the density $p_{i}$, we use the well-known formula (see, e.g., $\left.[4,13]\right)$ :

$$
\left(A \nabla H_{i}(x) \cdot n(x)\right)^{ \pm}= \pm \frac{p_{i}(x)}{2}+\int_{\partial \omega} p_{i}(y)(A \nabla E(x-y) \cdot n(x)) \mathrm{d} s(y) .
$$

Substituting these expressions into the fourth equation of (7.1) leads to the integral equation

$$
\left(\alpha_{1}+\alpha_{0}\right) \frac{p_{i}(x)}{2}+\left(\alpha_{1}-\alpha_{0}\right) \int_{\partial \omega} p_{i}(y)\left(A \nabla H_{i}(x) \cdot n(x)\right) \mathrm{d} s(y)=\left(\alpha_{1}-\alpha_{0}\right)(A n(x))_{i} \quad \forall x \in \partial \omega .
$$

When $\alpha_{1} \neq \alpha_{0}$, the above equation is equivalent to (4.2) with $r=\frac{\alpha_{1}}{\alpha_{0}}$. When $\alpha_{1}=\alpha_{0}$, we get $p_{i}=0$ and $H_{i}=0$. In particular, the following lemma holds with the convention ${\stackrel{\alpha_{0}}{\mathcal{P}}}_{\omega, 1}=0$.

Lemma 7.1. Let $H=\left(H_{1}, \ldots, H_{d}\right)^{\top}$ be the vector field defined as above and $k \in \mathbb{R}^{d}$. Then we have

$$
\left(\alpha_{1}-\alpha_{0}\right) \int_{\partial \omega}(A \nabla(H \cdot k) \cdot n)^{+} y \mathrm{~d} s(y)=-\alpha_{0} \mathcal{P}_{\omega, \frac{\alpha_{1}}{\alpha_{0}}} k+\left(\alpha_{1}-\alpha_{0}\right)|\omega| A k
$$

Proof. Let $I=\left(I_{1}, \ldots, I_{d}\right)^{\top}$ be the vector defined by

$$
I=\int_{\partial \omega}(A \nabla(H \cdot k) \cdot n)^{+} y \mathrm{~d} s(y)
$$

Then for each $j \in\{1, \ldots, d\}$, we have that

$$
I_{j}=\sum_{i} k_{i} \int_{\partial \omega}\left(A \nabla H_{i} \cdot n\right)^{+} y_{j} \mathrm{~d} s(y)
$$

Besides, from (7.4), we have the jump relation

$$
\left(A \nabla H_{i} \cdot n\right)^{+}-\left(A \nabla H_{i} \cdot n\right)^{-}=p_{i} .
$$


Combining (7.7) with the third equation of (7.1) brings

$$
\left(\alpha_{0}-\alpha_{1}\right)\left(A \nabla H_{i} \cdot n\right)^{+}=\alpha_{0} p_{i}-\left(\alpha_{1}-\alpha_{0}\right)(A n)_{i} .
$$

Equation (7.6) together with the above equality yield

$$
\left(\alpha_{1}-\alpha_{0}\right) I_{j}=\sum_{i} k_{i}\left[-\alpha_{0} \int_{\partial \omega} p_{i} y_{j} \mathrm{~d} s(y)+\left(\alpha_{1}-\alpha_{0}\right) \int_{\partial \omega}(A n)_{i} y_{j} \mathrm{~d} s(y)\right] .
$$

An integration by parts provides

$$
\int_{\partial \omega}(A n)_{i} y_{j} \mathrm{~d} s(y)=|\omega| A_{i j} .
$$

Gathering (7.9), (4.1) and (7.8) completes the proof.

For all $\varepsilon \in\left[0, \varepsilon_{0}\right)$ and for all $x \in \mathbb{R}^{d}$, we define the vector field $h_{\varepsilon}$ as

$$
h_{\varepsilon}(x)=\varepsilon H\left(\frac{x-x_{0}}{\varepsilon}\right) .
$$

Then, we have the following properties. We refer to [5] for the proof.

Lemma 7.2. Let $h_{\varepsilon}$ be the vector field defined as above and $R$ be a positive number. Then, for $\varepsilon$ going to zero, the following relations hold:

$$
\begin{aligned}
\left\|h_{\varepsilon}\right\|_{L^{2}(\Omega)} & =o\left(\varepsilon^{d / 2}\right), \\
\left\|\nabla h_{\varepsilon}\right\|_{L^{2}(\Omega)} & =O\left(\varepsilon^{d / 2}\right), \\
\left\|\nabla h_{\varepsilon}\right\|_{L^{2}\left(\Omega \backslash \overline{B\left(x_{0}, R\right)}\right)} & =O\left(\varepsilon^{d}\right) .
\end{aligned}
$$

\section{Asymptotic Behavior of the DiRECT AND ADJoint STATES}

We introduce the function

$$
\widehat{h}_{\varepsilon}(x, t)=-h_{\varepsilon}(x) \cdot \nabla v_{0}\left(x_{0}, t\right) \quad \forall(x, t) \in \mathbb{R}^{d} \times(0, T) .
$$

This function fulfills the following equations for all $t \in(0, T)$ :

$$
\left\{\begin{array}{cc}
\operatorname{div}\left(A \nabla \widehat{h}_{\varepsilon}(\cdot, t)\right)=0 & \text { in } \omega_{\varepsilon}, \\
\operatorname{div}\left(A \nabla \widehat{h}_{\varepsilon}(\cdot, t)\right)=0 & \text { in }\left(\mathbb{R}^{d} \backslash \overline{\omega_{\varepsilon}}\right), \\
\widehat{h}_{\varepsilon}^{+}(\cdot, t)=\widehat{h}_{\varepsilon}^{-}(\cdot, t) & \text { on } \partial \omega_{\varepsilon}, \\
\alpha_{1}\left(A \nabla \widehat{h}_{\varepsilon}(\cdot, t) \cdot n\right)^{+}-\alpha_{0}\left(A \nabla \widehat{h}_{\varepsilon}(\cdot, t) \cdot n\right)^{-}=-\left(\alpha_{1}-\alpha_{0}\right)\left(A \nabla v_{0}\left(x_{0}, t\right) \cdot n\right) & \text { on } \partial \omega_{\varepsilon}, \\
\widehat{h}_{\varepsilon}(\cdot, t) \rightarrow 0 & \text { at } \infty .
\end{array}\right.
$$

Furthermore, let us consider the function $e_{\varepsilon}$ such that

$$
v_{\varepsilon}=v_{0}+\widehat{h}_{\varepsilon}+e_{\varepsilon} .
$$

With the above notations, we have the following estimate whose proof is presented at the end of this section.

Lemma 8.1. The function $e_{\varepsilon}$ defined as above satisfies

$$
\left\|e_{\varepsilon}\right\|_{L^{\infty}\left(0, T ; L^{2}(\Omega)\right)}+\left\|e_{\varepsilon}\right\|_{L^{2}\left(0, T ; H^{1}(\Omega)\right)}=o\left(\varepsilon^{d / 2}\right) .
$$


As a consequence of the above lemma and of Lemma 7.2 we have the following result.

Lemma 8.2. Let $v_{\varepsilon}$ and $v_{0}$ be defined by (2.10). Consider a positive number $R$. Then, we have the following relations

$$
\begin{aligned}
\left\|v_{\varepsilon}-v_{0}\right\|_{L^{\infty}\left(0, T ; L^{2}(\Omega)\right)} & =o\left(\varepsilon^{d / 2}\right), \\
\left\|v_{\varepsilon}-v_{0}\right\|_{L^{2}\left(0, T ; H^{1}(\Omega)\right)} & =O\left(\varepsilon^{d / 2}\right), \\
\left\|\nabla\left(v_{\varepsilon}-v_{0}\right)\right\|_{L^{2}\left(0, T ; L^{2}\left(\Omega \backslash \overline{B\left(x_{0}, R\right)}\right)\right)} & =o\left(\varepsilon^{d / 2}\right) .
\end{aligned}
$$

We also have the corresponding result on the direct state. Indeed, it solves a similar PDE with a right hand side whose variation also satisfies $\left\|F_{\varepsilon}-F_{0}\right\|_{L^{2}\left(0, T ; H^{-1}(\Omega)\right)}=o\left(\varepsilon^{d / 2}\right)$. This latter statement is a straightforward consequence of (3.6).

Lemma 8.3. Let $u_{\varepsilon}$ and $u_{0}$ be defined by (2.1). Consider a positive number $R$. Then, we have the following relations

$$
\begin{aligned}
\left\|u_{\varepsilon}-u_{0}\right\|_{L^{\infty}\left(0, T ; L^{2}(\Omega)\right)} & =o\left(\varepsilon^{d / 2}\right), \\
\left\|u_{\varepsilon}-u_{0}\right\|_{L^{2}\left(0, T ; H^{1}(\Omega)\right)} & =O\left(\varepsilon^{d / 2}\right), \\
\left\|\nabla\left(u_{\varepsilon}-u_{0}\right)\right\|_{L^{2}\left(0, T ; L^{2}\left(\Omega \backslash \overline{B\left(x_{0}, R\right)}\right)\right)} & =o\left(\varepsilon^{d / 2}\right) .
\end{aligned}
$$

Proof of Lemma 8.1. Using (2.11) and (8.2) and the fact that $\widehat{h}_{\varepsilon}(\cdot, T)=0$, we easily check that $e_{\varepsilon}$ solves

$$
\left\{\begin{aligned}
-\rho_{1} \frac{\partial e_{\varepsilon}}{\partial t}-\alpha_{1} \operatorname{div}\left(A \nabla e_{\varepsilon}\right) & =Q_{1}+Q_{2}+Q_{3}+Q_{4} & & \text { in } \omega_{\varepsilon} \times(0, T), \\
-\rho_{0} \frac{\partial e_{\varepsilon}}{\partial t}-\alpha_{0} \operatorname{div}\left(A \nabla e_{\varepsilon}\right) & =Q_{1}+Q_{4} & & \text { in }\left(\Omega \backslash \overline{\omega_{\varepsilon}}\right) \times(0, T), \\
e_{\varepsilon}^{+} & =e_{\varepsilon}^{-} & & \text {on } \partial \omega_{\varepsilon} \times(0, T), \\
\alpha_{1}\left(A \nabla e_{\varepsilon} \cdot n\right)^{+}-\alpha_{0}\left(A \nabla e_{\varepsilon} \cdot n\right)^{-} & =Q_{5} & & \text { on } \partial \omega_{\varepsilon} \times(0, T), \\
e_{\varepsilon} & =-\widehat{h}_{\varepsilon} & & \text { on } \partial \Omega \times(0, T), \\
e_{\varepsilon}(\cdot, T) & =0 & & \text { in } \Omega,
\end{aligned}\right.
$$

where

$$
\begin{gathered}
Q_{1}=D J_{0}\left(u_{0}\right)-D J_{\varepsilon}\left(u_{0}\right), \quad Q_{2}=\left(\rho_{1}-\rho_{0}\right) \frac{\partial v_{0}}{\partial t}, \\
Q_{3}=\left(\alpha_{1}-\alpha_{0}\right) \operatorname{div}\left(A \nabla v_{0}\right), \quad Q_{4}=\rho_{\varepsilon} \frac{\partial \widehat{h}_{\varepsilon}}{\partial t}
\end{gathered}
$$

and for all $(x, t) \in \partial \omega_{\varepsilon} \times(0, T)$,

$$
Q_{5}(x, t)=-\left(\alpha_{1}-\alpha_{0}\right)\left(A\left[\nabla v_{0}(x, t)-\nabla v_{0}\left(x_{0}, t\right)\right] \cdot n\right) .
$$

In order to separate difficulties, we make the splitting

$$
e_{\varepsilon}=e_{1, \varepsilon}+e_{2, \varepsilon}
$$


with

and

$$
\left\{\begin{aligned}
-\rho_{1} \frac{\partial e_{1, \varepsilon}}{\partial t}-\alpha_{1} \operatorname{div}\left(A \nabla e_{1, \varepsilon}\right) & =Q_{1}+Q_{2}+Q_{3}+Q_{4} & & \text { in } \omega_{\varepsilon} \times(0, T), \\
-\rho_{0} \frac{\partial e_{1, \varepsilon}}{\partial t}-\alpha_{0} \operatorname{div}\left(A \nabla e_{1, \varepsilon}\right) & =Q_{1}+Q_{4} & & \text { in }\left(\Omega \backslash \overline{\omega_{\varepsilon}}\right) \times(0, T), \\
e_{1, \varepsilon}^{+} & =e_{1, \varepsilon}^{-} & & \text {on } \partial \omega_{\varepsilon} \times(0, T), \\
\alpha_{1}\left(A \nabla e_{1, \varepsilon} \cdot n\right)^{+}-\alpha_{0}\left(A \nabla e_{1, \varepsilon} \cdot n\right)^{-} & =Q_{5} & & \text { on } \partial \omega_{\varepsilon} \times(0, T), \\
e_{1, \varepsilon} & =0 & & \text { on } \partial \Omega \times(0, T), \\
e_{1, \varepsilon}(\cdot, T) & =0 & & \text { in } \Omega,
\end{aligned}\right.
$$

$$
\left\{\begin{aligned}
-\rho_{\varepsilon} \frac{\partial e_{2, \varepsilon}}{\partial t}-\operatorname{div}\left(\alpha_{\varepsilon} A \nabla e_{2, \varepsilon}\right) & =0 & & \text { in } \Omega \times(0, T), \\
e_{2, \varepsilon} & =-\widehat{h}_{\varepsilon} & & \text { on } \partial \Omega \times(0, T), \\
e_{2, \varepsilon}(\cdot, T) & =0 & & \text { in } \Omega .
\end{aligned}\right.
$$

We estimate $e_{1, \varepsilon}$ by multiplying the first two equations of (8.12) by $e_{1, \varepsilon}$ and by integrating in space and time:

$$
\begin{gathered}
\frac{1}{2} \int_{\Omega} \rho_{\varepsilon}\left|e_{1, \varepsilon}\left(\cdot, t_{0}\right)\right|^{2} \mathrm{~d} x+\int_{t_{0}}^{T} \int_{\Omega} \alpha_{\varepsilon} A \nabla e_{1, \varepsilon} \cdot \nabla e_{1, \varepsilon} \mathrm{d} x \mathrm{~d} t \leq \int_{t_{0}}^{T}\left|\int_{\partial \omega_{\varepsilon}} Q_{5} e_{1, \varepsilon} \mathrm{d} s\right| \mathrm{d} t+\left(\left\|Q_{1}\right\|_{L^{2}\left(t_{0}, T ; H^{-1}(\Omega)\right)}\right. \\
\left.+\left\|Q_{2} \chi_{\omega_{\varepsilon}}\right\|_{L^{2}\left(t_{0}, T ; H^{-1}(\Omega)\right)}+\left\|Q_{3} \chi_{\omega_{\varepsilon}}\right\|_{L^{2}\left(t_{0}, T ; H^{-1}(\Omega)\right)}+\left\|Q_{4}\right\|_{L^{2}\left(t_{0}, T ; H^{-1}(\Omega)\right)}\right)\left\|e_{1, \varepsilon}\right\|_{L^{2}\left(t_{0}, T ; H_{0}^{1}(\Omega)\right)},
\end{gathered}
$$

for almost all $t_{0} \in[0, T]$. Here, $\chi_{\omega_{\varepsilon}}$ stands for the characteristic function of the set $\omega_{\varepsilon}$.

Using the Poincaré inequality and taking the supremum for $t_{0} \in[0, T]$, the above equation yields

$$
\begin{aligned}
& \left\|e_{1, \varepsilon}\right\|_{L^{\infty}\left(0, T ; L^{2}(\Omega)\right)}^{2}+\left\|e_{1, \varepsilon}\right\|_{L^{2}\left(0, T ; H^{1}(\Omega)\right)}^{2} \leq C \int_{0}^{T}\left|\int_{\partial \omega_{\varepsilon}} Q_{5} e_{1, \varepsilon} \mathrm{d} s\right| \mathrm{d} t+C\left(\left\|Q_{1}\right\|_{L^{2}\left(0, T ; H^{-1}(\Omega)\right)}\right. \\
& \left.+\left\|Q_{2} \chi_{\omega_{\varepsilon}}\right\|_{L^{2}\left(0, T ; H^{-1}(\Omega)\right)}+\left\|Q_{3} \chi_{\omega_{\varepsilon}}\right\|_{L^{2}\left(0, T ; H^{-1}(\Omega)\right)}+\left\|Q_{4}\right\|_{L^{2}\left(0, T ; H^{-1}(\Omega)\right)}\right)\left\|e_{1, \varepsilon}\right\|_{L^{2}\left(0, T ; H_{0}^{1}(\Omega)\right)}
\end{aligned}
$$

Here and in the sequel, $C$ is used to denote any constant (independent of $\varepsilon$ ), that may change from place to place. Using the regularity of $\nabla v_{0}$ and the change of variables $x=x_{0}+\varepsilon y$, we obtain that

$$
\int_{0}^{T}\left|\int_{\partial \omega_{\varepsilon}} Q_{5} e_{1, \varepsilon} \mathrm{d} s\right| \mathrm{d} t \leq C \varepsilon^{d}\left\|v_{0}\right\|_{L^{2}\left(0, T ; W^{2, \infty}(\tilde{\Omega})\right)}\left(\int_{0}^{T} \int_{\partial \omega}\left|e_{1, \varepsilon}(\varepsilon y, t)\right|^{2} \mathrm{~d} s(y) \mathrm{d} t\right)^{1 / 2} .
$$

By the trace theorem and the change of variables $y=\varepsilon^{-1}\left(x-x_{0}\right)$, it comes

$$
\int_{0}^{T} \int_{\partial \omega}\left|e_{1, \varepsilon}(\varepsilon y, t)\right|^{2} \mathrm{~d} s(y) \mathrm{d} t \leq C \int_{0}^{T}\left(\varepsilon^{-d}\left\|e_{1, \varepsilon}\right\|_{L^{2}\left(\omega_{\varepsilon}\right)}^{2}+\varepsilon^{2-d}\left\|\nabla e_{1, \varepsilon}\right\|_{L^{2}\left(\omega_{\varepsilon}\right)}^{2}\right) \mathrm{d} t
$$

Hence, using the Sobolev inclusion $H^{1}(\Omega) \subset L^{6}(\Omega)$ (since $d=2$ or 3 ) and the Hölder inequality, we obtain that

$$
\int_{0}^{T} \int_{\partial \omega}\left|e_{1, \varepsilon}(\varepsilon y, t)\right|^{2} \mathrm{~d} s(y) \mathrm{d} t \leq C \int_{0}^{T}\left(\varepsilon^{-d / 3}\left\|e_{1, \varepsilon}\right\|_{H^{1}(\Omega)}^{2}+\varepsilon^{2-d}\left\|\nabla e_{1, \varepsilon}\right\|_{L^{2}\left(\omega_{\varepsilon}\right)}^{2}\right) \mathrm{d} t .
$$

From (8.16) and the above equation, it follows

$$
\int_{0}^{T}\left|\int_{\partial \omega_{\varepsilon}} Q_{5} e_{1, \varepsilon} \mathrm{d} s\right| \mathrm{d} t \leq C \varepsilon^{\frac{5 d}{6}}\left\|v_{0}\right\|_{L^{2}\left(0, T ; W^{2, \infty}(\tilde{\Omega})\right)}\left\|e_{1, \varepsilon}\right\|_{L^{2}\left(0, T ; H^{1}(\Omega)\right)} .
$$


Applying Lemma 7.2 leads to the following estimate on $Q_{4}$ :

$$
\left\|Q_{4}\right\|_{L^{2}\left(0, T ; H^{-1}(\Omega)\right)} \leqslant C\left\|\nabla v_{0}\left(x_{0}, \cdot\right)\right\|_{H^{1}(0, T)}\left\|h_{\varepsilon}\right\|_{H^{-1}(\Omega)}=o\left(\varepsilon^{d / 2}\right)\left\|\nabla v_{0}\left(x_{0}, \cdot\right)\right\|_{H^{1}(0, T)} .
$$

The Sobolev imbedding $L^{6 / 5}(\Omega) \subset H^{-1}(\Omega)$ (since $d=2$ or 3 ) leads to the inequalities

$$
\left\|Q_{2} \chi_{\omega_{\varepsilon}}\right\|_{L^{2}\left(0, T ; H^{-1}(\Omega)\right)} \leqslant\left\|Q_{2} \chi_{\omega_{\varepsilon}}\right\|_{L^{2}\left(0, T ; L^{6 / 5}(\Omega)\right)} \leqslant C\left\|\frac{\partial v_{0}}{\partial t}\right\|_{L^{2}\left(0, T ; L^{\infty}(\tilde{\Omega})\right)} \varepsilon^{5 d / 6}
$$

and

$$
\left\|Q_{3} \chi_{\omega_{\varepsilon}}\right\|_{L^{2}\left(0, T ; H^{-1}(\Omega)\right)} \leqslant C\left\|Q_{3} \chi_{\omega_{\varepsilon}}\right\|_{L^{2}\left(0, T ; L^{6 / 5}(\Omega)\right)} \leqslant C\left\|v_{0}\right\|_{L^{2}\left(0, T ; W^{2, \infty}(\tilde{\Omega})\right)} \varepsilon^{5 d / 6} .
$$

From (2.9), we have that

Gathering (8.15), (8.17)-(8.21), we obtain that

$$
\left\|Q_{1}\right\|_{L^{2}\left(0, T ; H^{-1}(\Omega)\right)}=o\left(\varepsilon^{d / 2}\right) .
$$

$$
\left\|e_{1, \varepsilon}\right\|_{L^{\infty}\left(0, T ; L^{2}(\Omega)\right)}^{2}+\left\|e_{1, \varepsilon}\right\|_{L^{2}\left(0, T ; H^{1}(\Omega)\right)}^{2} \leq o\left(\varepsilon^{d / 2}\right)\left\|e_{1, \varepsilon}\right\|_{L^{2}\left(0, T ; H^{1}(\Omega)\right)}
$$

which, combined with the Young inequality, provides

$$
\left\|e_{1, \varepsilon}\right\|_{L^{\infty}\left(0, T ; L^{2}(\Omega)\right)}+\left\|e_{1, \varepsilon}\right\|_{L^{2}\left(0, T ; H^{1}(\Omega)\right)}=o\left(\varepsilon^{d / 2}\right) .
$$

In order to estimate $e_{2, \varepsilon}$, we consider a smooth function $\theta: \Omega \rightarrow \mathbb{R}$ such that $\theta=0$ in $B\left(x_{0}, R\right)$ and $\theta=1$ on $\partial \Omega$. Then we set

$$
\begin{gathered}
\widetilde{h}_{\varepsilon}(x, t)=\widehat{h}_{\varepsilon}(x, t) \theta(x), \\
\widetilde{e}_{2, \varepsilon}(x, t)=e_{2, \varepsilon}(x, t)+\widetilde{h}_{\varepsilon}(x, t) .
\end{gathered}
$$

The function $\widetilde{e}_{2, \varepsilon}$ solves

$$
\left\{\begin{aligned}
-\rho_{\varepsilon} \frac{\partial \widetilde{e}_{2, \varepsilon}}{\partial t}-\operatorname{div}\left(\alpha_{\varepsilon} A \nabla \widetilde{e}_{2, \varepsilon}\right) & =-\rho_{\varepsilon} \frac{\partial \widetilde{h}_{\varepsilon}}{\partial t}-\operatorname{div}\left(\alpha_{\varepsilon} A \nabla \widetilde{h}_{\varepsilon}\right) & & \text { in } \Omega \times(0, T), \\
\widetilde{e}_{2, \varepsilon} & =0 & & \text { on } \partial \Omega \times(0, T), \\
\widetilde{e}_{2, \varepsilon}(\cdot, T) & =0 & & \text { in } \Omega .
\end{aligned}\right.
$$

By multiplying by $\widetilde{e}_{2, \varepsilon}$ and integrating by part, we obtain

$$
\left\|\widetilde{e}_{2, \varepsilon}\right\|_{L^{\infty}\left(0, T ; L^{2}(\Omega)\right)}+\left\|\widetilde{e}_{2, \varepsilon}\right\|_{L^{2}\left(0, T ; H^{1}(\Omega)\right)} \leq C\left(\left\|\frac{\partial \widetilde{h}_{\varepsilon}}{\partial t}\right\|_{L^{2}\left(0, T ; L^{2}(\Omega)\right)}+\left\|\widetilde{h}_{\varepsilon}\right\|_{L^{2}\left(0, T ; H^{1}(\Omega)\right)}\right) .
$$

From (8.24), (8.26), (8.23) and (8.1), successively, it comes:

$$
\begin{aligned}
\left\|e_{2, \varepsilon}\right\|_{L^{2}\left(0, T ; H^{1}(\Omega)\right)}+\left\|e_{2, \varepsilon}\right\|_{L^{\infty}\left(0, T ; L^{2}(\Omega)\right)} \leq & \left\|\tilde{e}_{2, \varepsilon}\right\|_{L^{2}\left(0, T ; H^{1}(\Omega)\right)}+\left\|\tilde{e}_{2, \varepsilon}\right\|_{L^{\infty}\left(0, T ; L^{2}(\Omega)\right)} \\
& +\left\|\tilde{h}_{\varepsilon}\right\|_{L^{2}\left(0, T ; H^{1}(\Omega)\right)}+\left\|\tilde{h}_{\varepsilon}\right\|_{L^{\infty}\left(0, T ; L^{2}(\Omega)\right)} \\
\leq & C\left(\left\|\tilde{h}_{\varepsilon}\right\|_{H^{1}\left(0, T ; L^{2}(\Omega)\right.}+\left\|\tilde{h}_{\varepsilon}\right\|_{L^{2}\left(0, T ; H^{1}(\Omega)\right)}\right) \\
\leq & C\left\|\nabla v_{0}\left(x_{0}, \cdot\right)\right\|_{H^{1}(0, T)}\left\|h_{\varepsilon}\right\|_{H^{1}\left(\Omega \backslash \overline{B\left(x_{0}, R\right)}\right)} .
\end{aligned}
$$

Then using Lemma 7.2 we derive

$$
\left\|e_{2, \varepsilon}\right\|_{L^{\infty}\left(0, T ; L^{2}(\Omega)\right)}+\left\|e_{2, \varepsilon}\right\|_{L^{2}\left(0, T ; H^{1}(\Omega)\right)}=o\left(\varepsilon^{d / 2}\right) .
$$

Combining (8.22) and (8.27) yields (8.4). 


\section{VARIATION OF THE BILINEAR FORM}

This section is devoted to the proof of Lemma 4.1. We study the behavior of the following quantity:

$$
\int_{0}^{T}\left(a_{\varepsilon}-a_{0}\right)\left(u_{0}, v_{\varepsilon}\right) \mathrm{d} t=\int_{0}^{T} \int_{\omega_{\varepsilon}}\left(\alpha_{1}-\alpha_{0}\right) A \nabla u_{0} \cdot \nabla v_{\varepsilon} \mathrm{d} x \mathrm{~d} t
$$

Adopting the decomposition (8.3), we write

$$
\begin{aligned}
\int_{0}^{T}\left(a_{\varepsilon}-a_{0}\right)\left(u_{0}, v_{\varepsilon}\right) \mathrm{d} t=\int_{0}^{T} \int_{\omega_{\varepsilon}}\left(\alpha_{1}-\alpha_{0}\right) A \nabla u_{0} \cdot \nabla v_{0} \mathrm{~d} x \mathrm{~d} t+\int_{0}^{T} & \int_{\omega_{\varepsilon}}\left(\alpha_{1}-\alpha_{0}\right) A \nabla u_{0} \cdot \nabla \widehat{h}_{\varepsilon} \mathrm{d} x \mathrm{~d} t \\
& +\int_{0}^{T} \int_{\omega_{\varepsilon}}\left(\alpha_{1}-\alpha_{0}\right) A \nabla u_{0} \cdot \nabla e_{\varepsilon} \mathrm{d} x \mathrm{~d} t .
\end{aligned}
$$

We shall prove later that:

$$
\begin{aligned}
\int_{0}^{T} \int_{\omega_{\varepsilon}}\left(\alpha_{1}-\alpha_{0}\right) A \nabla u_{0} \cdot \nabla \widehat{h}_{\varepsilon} \mathrm{d} x \mathrm{~d} t=\varepsilon^{d} \alpha_{0} \int_{0}^{T} & \nabla u_{0}\left(x_{0}, t\right) \cdot \mathcal{P}_{\omega, \frac{\alpha_{1}}{\alpha_{0}}} \nabla v_{0}\left(x_{0}, t\right) \mathrm{d} t \\
& -\varepsilon^{d}|\omega|\left(\alpha_{1}-\alpha_{0}\right) \int_{0}^{T} A \nabla u_{0}\left(x_{0}, t\right) \cdot \nabla v_{0}\left(x_{0}, t\right) \mathrm{d} t+o\left(\varepsilon^{d}\right) .
\end{aligned}
$$

Besides, we deduce from (8.4) and the Cauchy-Schwarz inequality, that

$$
\int_{0}^{T} \int_{\omega_{\varepsilon}}\left(\alpha_{1}-\alpha_{0}\right) A \nabla u_{0} \cdot \nabla e_{\varepsilon} \mathrm{d} x \mathrm{~d} t=\left\|\nabla u_{0}\right\|_{L^{2}\left(0, T ; L^{\infty}(\tilde{\Omega})\right)} o\left(\varepsilon^{d}\right),
$$

and from the regularity of $u_{0}$ and $v_{0}$, that

$$
\begin{array}{r}
\left|\int_{0}^{T} \int_{\omega_{\varepsilon}}\left(\alpha_{1}-\alpha_{0}\right) A \nabla u_{0} \cdot \nabla v_{0} \mathrm{~d} x \mathrm{~d} t-\varepsilon^{d}\right| \omega\left|\int_{0}^{T}\left(\alpha_{1}-\alpha_{0}\right) A \nabla u_{0}\left(x_{0}, t\right) \cdot \nabla v_{0}\left(x_{0}, t\right) \mathrm{d} t\right| \\
\leqslant C \varepsilon^{d+1}\left\|u_{0}\right\|_{L^{2}\left(0, T ; W^{2, \infty}(\tilde{\Omega})\right)}\left\|v_{0}\right\|_{L^{2}\left(0, T ; W^{2, \infty}(\tilde{\Omega})\right)} .
\end{array}
$$

Gathering (9.2)-(9.5) leads to Lemma 4.1.

It remains to prove (9.3). We recall that

$$
\widehat{h}_{\varepsilon}(x, t)=-\varepsilon H\left(\frac{x-x_{0}}{\varepsilon}\right) \cdot \nabla v_{0}\left(x_{0}, t\right) .
$$

Starting from the relation

$$
\int_{0}^{T} \int_{\omega_{\varepsilon}}\left(\alpha_{1}-\alpha_{0}\right) A \nabla u_{0} \cdot \nabla \widehat{h}_{\varepsilon} \mathrm{d} x \mathrm{~d} t=\int_{0}^{T} \int_{\omega_{\varepsilon}}\left(\alpha_{1}-\alpha_{0}\right) A \nabla\left(u_{0}(x, t)-u_{0}\left(x_{0}, t\right)\right) \cdot \nabla \widehat{h}_{\varepsilon}(x, t) \mathrm{d} x \mathrm{~d} t
$$

integrating by parts and using (8.2), we obtain

$$
\int_{0}^{T} \int_{\omega_{\varepsilon}}\left(\alpha_{1}-\alpha_{0}\right) A \nabla u_{0} \cdot \nabla \widehat{h}_{\varepsilon} \mathrm{d} x \mathrm{~d} t=\int_{0}^{T} \int_{\partial \omega_{\varepsilon}}\left(\alpha_{1}-\alpha_{0}\right)\left(u_{0}(x, t)-u_{0}\left(x_{0}, t\right)\right)\left(A \nabla \widehat{h}_{\varepsilon}(x, t) \cdot n\right)^{+} \mathrm{d} s(x) \mathrm{d} t .
$$


Using the change of variables $x=x_{0}+\varepsilon y$, we proceed by

$$
\begin{aligned}
\int_{0}^{T} \int_{\omega_{\varepsilon}}\left(\alpha_{1}-\alpha_{0}\right) A & \nabla u_{0} \cdot \nabla \widehat{h}_{\varepsilon} \mathrm{d} x \mathrm{~d} t \\
& =-\varepsilon^{d-1}\left(\alpha_{1}-\alpha_{0}\right) \int_{0}^{T} \int_{\partial \omega}\left(u_{0}\left(x_{0}+\varepsilon y, t\right)-u_{0}\left(x_{0}, t\right)\right)\left(A \nabla\left(H(y) \cdot \nabla v_{0}\left(x_{0}, t\right)\right) \cdot n\right)^{+} \mathrm{d} s(y) \mathrm{d} t .
\end{aligned}
$$

The regularity of $u_{0}$ leads to

$$
\int_{0}^{T} \int_{\omega_{\varepsilon}}\left(\alpha_{1}-\alpha_{0}\right) A \nabla u_{0} \cdot \nabla \widehat{h}_{\varepsilon} \mathrm{d} x \mathrm{~d} t=-\varepsilon^{d}\left(\alpha_{1}-\alpha_{0}\right) \int_{0}^{T} \nabla u_{0}\left(x_{0}, t\right) \cdot \int_{\partial \omega}\left(A \nabla\left(H(y) \cdot \nabla v_{0}\left(x_{0}, t\right)\right) \cdot n\right)^{+} y \mathrm{~d} s(y) \mathrm{d} t+o\left(\varepsilon^{d}\right) .
$$

Finally, applying Lemma 7.1 yields (9.3).

\section{VARIATION OF THE TERM INVOLVING THE TIME DERIVATIVE}

This section is devoted to the proof of Lemma 4.2. First we have that

$$
\int_{0}^{T}\left\langle\left(\rho_{\varepsilon}-\rho_{0}\right) \frac{\partial u_{0}}{\partial t}, v_{\varepsilon}\right\rangle_{H^{-1}(\Omega), H_{0}^{1}(\Omega)} \mathrm{d} t=\int_{0}^{T} \int_{\omega_{\varepsilon}}\left(\rho_{1}-\rho_{0}\right) \frac{\partial u_{0}}{\partial t} v_{\varepsilon} \mathrm{d} x \mathrm{~d} t
$$

and thus, we can write

$$
\int_{0}^{T}\left\langle\left(\rho_{\varepsilon}-\rho_{0}\right) \frac{\partial u_{0}}{\partial t}, v_{\varepsilon}\right\rangle_{H^{-1}(\Omega), H_{0}^{1}(\Omega)} \mathrm{d} t=\varepsilon^{d}\left(\rho_{1}-\rho_{0}\right)|\omega| \int_{0}^{T} \frac{\partial u_{0}}{\partial t}\left(x_{0}, t\right) v_{0}\left(x_{0}, t\right) \mathrm{d} t+S_{1}+S_{2},
$$

where

$$
\begin{gathered}
S_{1}=\int_{0}^{T} \int_{\omega_{\varepsilon}}\left(\rho_{1}-\rho_{0}\right) \frac{\partial u_{0}}{\partial t}\left(v_{\varepsilon}-v_{0}\right) \mathrm{d} x \mathrm{~d} t \\
S_{2}=\int_{0}^{T} \int_{\omega_{\varepsilon}}\left(\rho_{1}-\rho_{0}\right)\left[\frac{\partial u_{0}}{\partial t}(x, t) v_{0}(x, t)-\frac{\partial u_{0}}{\partial t}\left(x_{0}, t\right) v_{0}\left(x_{0}, t\right)\right] \mathrm{d} x \mathrm{~d} t .
\end{gathered}
$$

It stems from the regularity assumptions on $u_{0}$ and $v_{0}$ that

$$
\left|S_{2}\right| \leq C \varepsilon^{d+1}\left\|u_{0}\right\|_{H^{1}\left(0, T ; W^{1, \infty}(\widetilde{\Omega})\right)}\left\|v_{0}\right\|_{L^{2}\left(0, T ; W^{1, \infty}(\widetilde{\Omega})\right)} .
$$

Moreover, by using the Cauchy-Schwarz inequality in time and the Hölder inequality in space together with the imbedding $H^{1}(\Omega) \subset L^{6}(\Omega)$, it comes

$$
\left|S_{1}\right| \leq C \varepsilon^{5 d / 6}\left\|u_{0}\right\|_{H^{1}\left(0, T ; L^{\infty}(\tilde{\Omega})\right)}\left\|v_{\varepsilon}-v_{0}\right\|_{L^{2}\left(0, T ; H^{1}(\tilde{\Omega})\right)} .
$$

Applying (8.6), it follows

$$
\left|S_{1}\right|=O\left(\varepsilon^{4 d / 3}\right),
$$

which completes the proof. 


\section{VARIATION OF THE LINEAR FORM}

We turn to the variation

$$
\int_{0}^{T}\left(\ell_{\varepsilon}-\ell_{0}\right)\left(v_{\varepsilon}\right) \mathrm{d} t=\int_{0}^{T} \int_{\omega_{\varepsilon}}\left(F_{1}-F_{0}\right) v_{\varepsilon} \mathrm{d} x \mathrm{~d} t
$$

We have that

$$
\int_{0}^{T}\left(\ell_{\varepsilon}-\ell_{0}\right)\left(v_{\varepsilon}\right) \mathrm{d} t=\varepsilon^{d}|\omega| \int_{0}^{T}\left(F_{1}\left(x_{0}, t\right)-F_{0}\left(x_{0}, t\right)\right) v_{0}\left(x_{0}, t\right) \mathrm{d} t+R_{1}+R_{2}
$$

where

$$
\begin{gathered}
R_{1}=\int_{0}^{T} \int_{\omega_{\varepsilon}}\left(F_{1}-F_{0}\right)\left(v_{\varepsilon}-v_{0}\right) \mathrm{d} x \mathrm{~d} t \\
R_{2}=\int_{0}^{T} \int_{\omega_{\varepsilon}}\left[\left(F_{1}(x, t)-F_{0}(x, t)\right) v_{0}(x, t)-\left(F_{1}\left(x_{0}, t\right)-F_{0}\left(x_{0}, t\right)\right) v_{0}\left(x_{0}, t\right)\right] \mathrm{d} x \mathrm{~d} t .
\end{gathered}
$$

Using the regularity assumptions on $F_{0}$ and $F_{1}$, we obtain that

$$
\left|R_{2}\right| \leqslant C \varepsilon^{d+1}\left(\left\|F_{1}\right\|_{L^{2}\left(0, T ; W^{1, \infty}(\tilde{\Omega})\right)}+\left\|F_{0}\right\|_{L^{2}\left(0, T ; W^{1, \infty}(\tilde{\Omega})\right)}\right)\left\|v_{0}\right\|_{L^{2}\left(0, T ; W^{1, \infty}(\tilde{\Omega})\right)} .
$$

Besides, thanks to the Cauchy-Schwarz inequality, we have

$$
\left|R_{1}\right| \leqslant C \varepsilon^{d / 2}\left(\left\|F_{1}\right\|_{L^{2}\left(0, T ; L^{\infty}(\tilde{\Omega})\right)}+\left\|F_{0}\right\|_{L^{2}\left(0, T ; L^{\infty}(\tilde{\Omega})\right)}\right)\left\|v_{0}-v_{\varepsilon}\right\|_{L^{2}\left(0, T ; L^{2}(\tilde{\Omega})\right)} .
$$

Hence, by using (8.5), we derive

$$
\left|R_{1}\right|=o\left(\varepsilon^{d}\right) .
$$

Gathering (11.1), (11.2) and (11.3), we obtain Lemma 4.3.

\section{VARIATION OF THE COST FUnCtional}

Proof of Theorem 5.1. First, since $J_{\varepsilon} \in \mathcal{C}\left(L^{2}(\Omega) ; \mathbb{R}\right)$, and

$$
X \subset \mathcal{C}\left([0, T] ; L^{2}(\Omega)\right),
$$

we have that for any $v \in X, J_{\varepsilon}(v):[0, T] \rightarrow \mathbb{R}$ is a continuous function. Therefore,

$$
\mathcal{J}_{\varepsilon}(v)=\int_{0}^{T} J_{\varepsilon}(v(t)) \mathrm{d} t
$$

is well-defined.

Now, we check (2.7) with $\delta \mathcal{J}_{1}=0$. We proceed by the Taylor formula:

$$
\begin{aligned}
\mathcal{J}_{\mathcal{E}}\left(u_{\varepsilon}\right)-\mathcal{J}_{\mathcal{\varepsilon}}\left(u_{0}\right)-\int_{0}^{T}\left\langle D J_{\varepsilon}\left(u_{0}(t)\right), u_{\varepsilon}(t)-u_{0}(t)\right\rangle_{H^{-1}(\Omega), H_{0}^{1}(\Omega)} \mathrm{d} t & \\
& =\frac{1}{2} \int_{0}^{T} D^{2} J_{\varepsilon}\left(w_{\varepsilon}(t)\right)\left(u_{\varepsilon}(t)-u_{0}(t), u_{\varepsilon}(t)-u_{0}(t)\right) \mathrm{d} t,
\end{aligned}
$$

where $w_{\varepsilon}(t) \in\left[u_{0}(t), u_{\varepsilon}(t)\right]$ for almost all $t \in[0, T]$. From Lemma 8.3, we have that

$$
\left\|u_{\varepsilon}(t)-u_{0}(t)\right\|_{L^{\infty}\left(0, T ; L^{2}(\Omega)\right)}=o\left(\varepsilon^{d / 2}\right),
$$


and thus

$$
\left\|w_{\varepsilon}(t)-u_{0}(t)\right\|_{L^{\infty}\left(0, T ; L^{2}(\Omega)\right)}=o\left(\varepsilon^{d / 2}\right) .
$$

Consequently, for some positive number $M$, we have

$$
\left\|w_{\varepsilon}(t)\right\|_{L^{2}(\Omega)} \leq M \quad \forall t \in[0, T] .
$$

From this bound together with (5.1), we derive that

$$
\left\|D^{2} J_{\varepsilon}\left(w_{\varepsilon}(t)\right)\right\|_{\mathcal{B}\left(L^{2}(\Omega)\right)} \leq C(M) \quad \forall t \in[0, T]
$$

which implies, by using (12.1),

$$
\left|\int_{0}^{T} D^{2} J_{\varepsilon}\left(w_{\varepsilon}(t)\right)\left(u_{\varepsilon}(t)-u_{0}(t), u_{\varepsilon}(t)-u_{0}(t)\right) \mathrm{d} t\right| \leq C(M) \int_{0}^{T}\left\|u_{\varepsilon}(t)-u_{0}(t)\right\|_{L^{2}(\Omega)}^{2} \mathrm{~d} t=o\left(\varepsilon^{d}\right) .
$$

Proof of Corollary 5.1.

(1) For the functional

$$
J_{\varepsilon}(u)=\int_{\Omega}\left|u-u_{d}\right|^{2} \mathrm{~d} x,
$$

it is obvious that $J_{\varepsilon} \in \mathcal{C}^{2}\left(L^{2}(\Omega), \mathbb{R}\right)$ and that (5.1) is satisfied, so that we can apply Theorem 5.1. Therefore (2.7) holds true. Since in this case $J_{\varepsilon}$ does not depend on $\varepsilon$, relations (2.8) and (2.9) (with $\delta \mathcal{J}_{2}=0$ ) hold true. The regularity condition (3.3) is also fulfilled since $u_{0}$ satisfies (3.4) and $u_{d} \in$ $H^{4}\left(B\left(x_{0}, R\right)\right)$. Therefore we can apply Theorem 4.1 and we obtain the asymptotic expansion (4.6).

(2) For the functional

$$
J_{\varepsilon}(u)=\int_{\Omega} \alpha_{\varepsilon}\left|u-u_{d}\right|^{2} \mathrm{~d} x,
$$

Theorem 5.1 can also be applied. Therefore (2.7) holds true. The condition (3.3) is fulfilled for the same reasons as before. Next, we have

$$
\mathcal{J}_{\varepsilon}\left(u_{0}\right)-\mathcal{J}_{0}\left(u_{0}\right)=\int_{0}^{T} \int_{\omega_{\varepsilon}}\left(\alpha_{1}-\alpha_{0}\right)\left|u_{0}(x, t)-u_{d}(x)\right|^{2} \mathrm{~d} x \mathrm{~d} t .
$$

From the regularity assumptions on $u_{0}$ and $u_{d}$, we have that

$$
\mathcal{J}_{\varepsilon}\left(u_{0}\right)-\mathcal{J}_{0}\left(u_{0}\right)=\int_{0}^{T}\left|\omega_{\varepsilon}\right|\left(\alpha_{1}-\alpha_{0}\right)\left|u_{0}\left(x_{0}, t\right)-u_{d}\left(x_{0}\right)\right|^{2} \mathrm{~d} t+O\left(\varepsilon^{d+1}\right),
$$

which implies (2.8). Finally, for any $\varphi \in L^{2}\left(0, T ; H_{0}^{1}(\Omega)\right)$,

$$
\int_{0}^{T}\left\langle D J_{\varepsilon}\left(u_{0}(t)\right)-D J_{0}\left(u_{0}(t)\right), \varphi(t)\right\rangle_{H^{-1}(\Omega), H_{0}^{1}(\Omega)} \mathrm{d} t=2 \int_{0}^{T} \int_{\omega_{\varepsilon}}\left(\alpha_{1}-\alpha_{0}\right)\left(u_{0}(x, t)-u_{d}(x)\right) \varphi(x, t) \mathrm{d} x \mathrm{~d} t .
$$

From the Cauchy-Schwarz inequality, it comes

$$
\begin{aligned}
& \int_{0}^{T}\left\langle D J_{\varepsilon}\left(u_{0}(t)\right)-D J_{0}\left(u_{0}(t)\right), \varphi(t)\right\rangle_{H^{-1}(\Omega), H_{0}^{1}(\Omega)} \mathrm{d} t \\
& \quad \leq C \int_{0}^{T}\left(\int_{\omega_{\varepsilon}}\left|u_{0}(x, t)-u_{d}(x)\right|^{2} \mathrm{~d} x\right)^{1 / 2}\left(\int_{\omega_{\varepsilon}}|\varphi(x, t)|^{2} \mathrm{~d} x\right)^{1 / 2} \mathrm{~d} t .
\end{aligned}
$$


Using the Sobolev embedding $H^{1}(\Omega) \subset L^{6}(\Omega)$ and the Hölder inequality, we obtain

$\int_{0}^{T}\left\langle D J_{\varepsilon}\left(u_{0}(t)\right)-D J_{0}\left(u_{0}(t)\right), \varphi(t)\right\rangle_{H^{-1}(\Omega), H_{0}^{1}(\Omega)} \mathrm{d} t \leq C \varepsilon^{2 d / 3} \int_{0}^{T}\left\|u_{0}(t)-u_{d}\right\|_{H^{1}(\Omega)}\|\varphi(t)\|_{H^{1}(\Omega)} \mathrm{d} t$.

Another application of the Cauchy-Schwarz inequality provides

$\int_{0}^{T}\left\langle D J_{\varepsilon}\left(u_{0}(t)\right)-D J_{0}\left(u_{0}(t)\right), \varphi(t)\right\rangle_{H^{-1}(\Omega), H_{0}^{1}(\Omega)} \mathrm{d} t \leq C \varepsilon^{2 d / 3}\left\|u_{0}-u_{d}\right\|_{L^{2}\left(0, T ; H^{1}(\Omega)\right)}\|\varphi\|_{L^{2}\left(0, T ; H^{1}(\Omega)\right)}$,

which leads to (2.9). The condition (3.3) can be checked in the same way as in the previous example.

Proof of Proposition 5.1.

(1) For the functional

$$
J_{\varepsilon}(u)=\int_{\Omega} \eta(x) A \nabla\left(u-u_{d}\right) \cdot \nabla\left(u-u_{d}\right) \mathrm{d} x,
$$

we easily see that $\mathcal{J}_{\varepsilon}$ is well-defined on $X$ and fulfills (2.8) with $\delta \mathcal{J}_{2}=0$. The condition (2.9) holds true since $J_{\varepsilon}$ does not depend on $\varepsilon$. Next we consider the variation

$\left|\mathcal{J}_{\mathcal{\varepsilon}}\left(u_{\varepsilon}\right)-\mathcal{J}_{\varepsilon}\left(u_{0}\right)-\int_{0}^{T}\left\langle D J_{\varepsilon}\left(u_{0}\right),\left(u_{\varepsilon}-u_{0}\right)\right\rangle_{H^{-1}(\Omega), H_{0}^{1}(\Omega)} \mathrm{d} t\right|=\int_{0}^{T} \int_{\Omega} \eta(x) A \nabla\left(u_{\varepsilon}-u_{0}\right) \cdot \nabla\left(u_{\varepsilon}-u_{0}\right) \mathrm{d} x \mathrm{~d} t$.

The above equation together with $(8.10)$ yield $\delta \mathcal{J}_{1}=0$. We now check (3.3). We have

$$
D J_{0}\left(u_{0}\right)=-2 \operatorname{div}\left(\eta A \nabla\left(u_{0}-u_{d}\right)\right)
$$

This function belongs to $L^{2}\left(0, T ; H^{4}\left(\Omega_{J}\right)\right) \cap H^{2}\left(0, T ; L^{2}\left(\Omega_{J}\right)\right)$ for any $\Omega_{J} \subset B\left(x_{0}, R\right)$.

(2) For the functional

$$
\mathcal{J}_{\varepsilon}(u)=\int_{0}^{T} \int_{\partial \Omega}\left|u-u_{d}\right|^{2} \mathrm{~d} s \mathrm{~d} t
$$

we easily check that $\mathcal{J}_{\varepsilon}$ is well-defined on $X$ and fulfills (2.8), (2.9) with $\delta \mathcal{J}_{2}=0$. We have that

$$
\left|\mathcal{J}_{\varepsilon}\left(u_{\varepsilon}\right)-\mathcal{J}_{\varepsilon}\left(u_{0}\right)-\int_{0}^{T}\left\langle D J_{\varepsilon}\left(u_{0}\right),\left(u_{\varepsilon}-u_{0}\right)\right\rangle_{\left(H^{1}(\Omega)\right)^{\prime}, H^{1}(\Omega)} \mathrm{d} t\right|=\int_{0}^{T} \int_{\partial \Omega}\left(u_{\varepsilon}-u_{0}\right)^{2} \mathrm{~d} x \mathrm{~d} t
$$

It follows from (8.8) and (8.10) that $\delta \mathcal{J}_{1}=0$. The adjoint state $v_{0}$ satisfies a non-homogeneous Neumann boundary condition with source term

$$
g=2\left(u_{0}-u_{d}\right) .
$$

The regularity $v_{0} \in L^{2}\left(0, T ; H^{6}\left(\widetilde{\Omega}_{J}\right)\right) \cap H^{3}\left(0, T ; L^{2}\left(\widetilde{\Omega}_{J}\right)\right)$, for some suitable $\widetilde{\Omega}_{J}$, stems from Lemma 6.1. 
Part 2. Topological sensitivity analysis for hyperbolic problems

\section{SEtTing OF the PROBlem}

With the same notations as before, we consider now the wave equation:

$$
\left\{\begin{aligned}
\rho_{\varepsilon} \frac{\partial^{2} u_{\varepsilon}}{\partial t^{2}}-\operatorname{div}\left(\alpha_{\varepsilon} A \nabla u_{\varepsilon}\right) & =F_{\varepsilon} & & \text { in } \Omega \times(0, T), \\
u_{\varepsilon} & =0 & & \text { on } \partial \Omega \times(0, T), \\
u_{\varepsilon}(\cdot, 0) & =\frac{\partial u_{\varepsilon}}{\partial t}(\cdot, 0)=0 & & \text { in } \Omega .
\end{aligned}\right.
$$

The corresponding variational formulation for

$$
\begin{gathered}
X=\mathcal{C}\left([0, T] ; H_{0}^{1}(\Omega)\right) \cap \mathcal{C}^{1}\left([0, T] ; L^{2}(\Omega)\right) \cap \mathcal{C}^{2}\left([0, T] ; H^{-1}(\Omega)\right), \\
u_{\varepsilon} \in X_{0}=\left\{u \in X, u(., 0)=\frac{\partial u_{\varepsilon}}{\partial t}(\cdot, 0)=0\right\}
\end{gathered}
$$

reads

$$
\int_{0}^{T}\left\langle\rho_{\varepsilon} \frac{\partial^{2} u_{\varepsilon}}{\partial t^{2}}, v\right\rangle_{H^{-1}(\Omega), H_{0}^{1}(\Omega)} \mathrm{d} t+\int_{0}^{T} a_{\varepsilon}\left(u_{\varepsilon}, v\right) \mathrm{d} t=\int_{0}^{T} \ell_{\varepsilon}(v) \mathrm{d} t \quad \forall v \in X,
$$

with the bilinear form $a_{\varepsilon}$ and the linear functional $\ell_{\varepsilon}$ defined by (2.3) and (2.4). We write (13.3) in the general form (1.1) by setting

$$
\begin{gathered}
\mathcal{A}_{\varepsilon}(u, v)=\int_{0}^{T}\left(\left\langle\rho_{\varepsilon} \frac{\partial^{2} u}{\partial t^{2}}, v\right\rangle_{H^{-1}(\Omega), H_{0}^{1}(\Omega)}+a_{\varepsilon}(u, v)\right) \mathrm{d} t, \\
\mathcal{L}_{\varepsilon}(v)=\int_{0}^{T} \ell_{\varepsilon}(v) \mathrm{d} t .
\end{gathered}
$$

We consider a cost functional of the form (2.5) satisfying (2.6), (2.7), (2.8) and such that

$$
\left\|D J_{0}\left(u_{0}\right)-D J_{\varepsilon}\left(u_{0}\right)\right\|_{W^{1,1}\left(0, T ; H^{-1}(\Omega)\right)}=o\left(\varepsilon^{d / 2}\right) .
$$

The adjoint state $v_{\varepsilon} \in X$ defined by (1.4) solves:

$$
\int_{0}^{T}\left\langle\rho_{\varepsilon} \frac{\partial^{2} \varphi}{\partial t^{2}}, v_{\varepsilon}\right\rangle_{H^{-1}(\Omega), H_{0}^{1}(\Omega)} \mathrm{d} t+\int_{0}^{T} a_{\varepsilon}\left(\varphi, v_{\varepsilon}\right) \mathrm{d} t=-\int_{0}^{T} D J_{\varepsilon}\left(u_{0}\right) \varphi \mathrm{d} t \quad \forall \varphi \in X_{0} .
$$

The associated strong formulation reads:

$$
\left\{\begin{array}{cl}
\rho_{\varepsilon} \frac{\partial^{2} v_{\varepsilon}}{\partial t^{2}}-\operatorname{div}\left(\alpha_{\varepsilon} A \nabla v_{\varepsilon}\right)=-D J_{\varepsilon}\left(u_{0}\right) & \text { in } \Omega \times(0, T), \\
v_{\varepsilon}=0 & \text { on } \partial \Omega \times(0, T), \\
v_{\varepsilon}(\cdot, T)=\frac{\partial v_{\varepsilon}}{\partial t}(\cdot, T)=0 & \text { in } \Omega .
\end{array}\right.
$$

\section{Regularity ASSUmptions}

For notational simplicity, we define the differential operator

$$
\Lambda: u \mapsto \operatorname{div}\left(\alpha_{0} A \nabla u\right)
$$


The needed regularity on the direct and adjoint solutions can be obtained from different sets of assumptions. The following one is chosen merely as an example:

$$
\begin{gathered}
F_{0} \in \mathcal{C}^{6}\left([0, T] ; L^{2}(\Omega)\right) \cap \bigcap_{j=0}^{4} \mathcal{C}^{j}\left([0, T] ; H^{5-j}(\Omega)\right), \\
F_{0}, \Lambda F_{0} \text { and } \Lambda^{2} F_{0} \text { vanish on } \partial \Omega, \\
F_{1} \in L^{2}\left(0, T ; W^{1, \infty}\left(B\left(x_{0}, R\right)\right), \quad R>0,\right. \\
D J_{0}\left(u_{0}\right) \in \mathcal{C}^{6}\left([0, T] ; L^{2}(\Omega)\right) \cap \bigcap_{j=0}^{4} \mathcal{C}^{j}\left([0, T] ; H^{5-j}(\Omega)\right), \\
D J_{0}\left(u_{0}\right), \Lambda D J_{0}\left(u_{0}\right) \text { and } \Lambda^{2} D J_{0}\left(u_{0}\right) \text { vanish on } \partial \Omega .
\end{gathered}
$$

The conditions (14.2)-(14.4) are assumed throughout all this part of the paper, whereas the conditions (14.5) and (14.6) will be checked later for some examples of cost functional. The following result is proved in Section 17.

Proposition 14.1. Assume that $u_{0}$ and $v_{0}$ solve (13.1) and (13.6), respectively, for $\varepsilon=0$, and that the regularity assumptions (14.2)-(14.6) hold. Then

$$
\begin{gathered}
u_{0} \in \mathcal{C}^{j}\left([0, T] ; H^{7-j}(\Omega)\right) \quad \forall j=0, \ldots, 7, \\
u_{0}, \Lambda u_{0} \text { and } \Lambda^{2} u_{0} \text { vanish on } \partial \Omega, \\
v_{0} \in \mathcal{C}^{j}\left([0, T] ; H^{7-j}(\Omega)\right) \quad \forall j=0, \ldots, 7, \\
v_{0}, \Lambda v_{0} \text { and } \Lambda^{2} v_{0} \text { vanish on } \partial \Omega .
\end{gathered}
$$

\section{MAin RESULT}

The following lemmas are proved in Section 17. The polarization matrix $\mathcal{P}_{\omega, \frac{\alpha_{1}}{\alpha_{0}}}$ involved in Lemma 15.1 is identical to that defined in the first part (see Sect. 4).

Lemma 15.1. Assume that the bilinear form $a_{\varepsilon}$ is defined by (2.3), that $u_{0}$ and $v_{\varepsilon}$ solve (13.1) and (13.6), respectively, that we have the regularity assumptions (14.2)-(14.6) and that (13.4) holds true.

Then we have

$$
\int_{0}^{T}\left(a_{\varepsilon}-a_{0}\right)\left(u_{0}, v_{\varepsilon}\right) \mathrm{d} t=\varepsilon^{d} \delta a+o\left(\varepsilon^{d}\right)
$$

with

$$
\delta a=\alpha_{0} \int_{0}^{T} \nabla u_{0}\left(x_{0}, t\right) \cdot \mathcal{P}_{\omega, \frac{\alpha_{1}}{\alpha_{0}}} \nabla v_{0}\left(x_{0}, t\right) \mathrm{d} t .
$$

Lemma 15.2. Assume that $u_{0}$ and $v_{\varepsilon}$ solve (13.1) and (13.6), respectively, that we have the regularity assumptions (14.2)-(14.6) and that (13.4) holds true.

Then, we have

$$
\int_{0}^{T}\left\langle\left(\rho_{\varepsilon}-\rho_{0}\right) \frac{\partial^{2} u_{0}}{\partial t^{2}}, v_{\varepsilon}\right\rangle_{H^{-1}(\Omega), H_{0}^{1}(\Omega)} \mathrm{d} t=\varepsilon^{d} \delta \rho+o\left(\varepsilon^{d}\right)
$$

with

$$
\delta \rho=-\left(\rho_{1}-\rho_{0}\right)|\omega| \int_{0}^{T} \frac{\partial u_{0}}{\partial t}\left(x_{0}, t\right) \frac{\partial v_{0}}{\partial t}\left(x_{0}, t\right) \mathrm{d} t .
$$


Lemma 15.3. Assume that the linear functional $\ell_{\varepsilon}$ is defined by (2.4), that $u_{0}$ and $v_{\varepsilon}$ solve (13.1) and (13.6), respectively, that we have the regularity assumptions (14.2)-(14.6) and that (13.4) holds true.

Then, we have

$$
\int_{0}^{T}\left(\ell_{\varepsilon}-\ell_{0}\right)\left(v_{\varepsilon}\right) \mathrm{d} t=\varepsilon^{d} \delta \ell+o\left(\varepsilon^{d}\right)
$$

with

$$
\delta \ell=|\omega| \int_{0}^{T}\left(F_{1}\left(x_{0}, t\right)-F_{0}\left(x_{0}, t\right)\right) v_{0}\left(x_{0}, t\right) \mathrm{d} t .
$$

As a consequence of Proposition 1.1 and the above lemmas, we obtain the following theorem.

Theorem 15.1. Assume that the cost functional $\mathcal{J}_{\varepsilon}$ satisfies (2.5)-(2.8) and (13.4). Suppose moreover that $u_{0}$ and $v_{0}$ solve (13.1) and (13.6), respectively, for $\varepsilon=0$, and that the regularity assumptions (14.2)-(14.6) hold. Then we have the following asymptotic expansion:

$$
\begin{array}{r}
j(\varepsilon)-j(0)=\varepsilon^{d}\left[-\left(\rho_{1}-\rho_{0}\right)|\omega| \int_{0}^{T} \frac{\partial u_{0}}{\partial t}\left(x_{0}, t\right) \frac{\partial v_{0}}{\partial t}\left(x_{0}, t\right) \mathrm{d} t+\alpha_{0} \int_{0}^{T} \nabla u_{0}\left(x_{0}, t\right) \cdot \mathcal{P}_{\omega, \frac{\alpha_{1}}{\alpha_{0}}} \nabla v_{0}\left(x_{0}, t\right) \mathrm{d} t\right. \\
\left.-|\omega| \int_{0}^{T}\left(F_{1}\left(x_{0}, t\right)-F_{0}\left(x_{0}, t\right)\right) v_{0}\left(x_{0}, t\right) \mathrm{d} t+\delta \mathcal{J}_{1}+\delta \mathcal{J}_{2}\right]+o\left(\varepsilon^{d}\right) .
\end{array}
$$

\section{Examples of COST FunCtional}

We consider the same examples as in the first part. The proofs, which are similar, are omitted.

Theorem 16.1. Theorem 5.1 is valid with the current notations, i.e. $X$ being defined by (13.2).

Corollary 16.1. The asymptotic expansion (15.4) holds true for the following cost functionals.

(1) For the functional

$$
J_{\varepsilon}(u)=\int_{\Omega}\left|u-u_{d}\right|^{2} \mathrm{~d} x
$$

with

$$
u_{d} \in H^{5}(\Omega) \text { and } u_{d}, \Lambda u_{d}, \Lambda^{2} u_{d} \text { vanishing on } \partial \Omega,
$$

the operator $\Lambda$ being defined by (14.1), we have $\delta \mathcal{J}_{1}=0$ and $\delta \mathcal{J}_{2}=0$. We recall that this functional cannot be considered in the case of a hole.

(2) For the functional

with

$$
J_{\varepsilon}(u)=\int_{\Omega} \alpha_{\varepsilon}\left|u-u_{d}\right|^{2} \mathrm{~d} x
$$

$$
u_{d} \in H^{5}(\Omega) \text { and } u_{d}, \Lambda u_{d}, \Lambda^{2} u_{d} \text { vanishing on } \partial \Omega,
$$

we have $\delta \mathcal{J}_{1}=0$ and

$$
\delta \mathcal{J}_{2}=\left(\alpha_{1}-\alpha_{0}\right)|\omega| \int_{0}^{T}\left|u_{0}\left(x_{0}, t\right)-u_{d}\left(x_{0}\right)\right| \mathrm{d} t .
$$

Proposition 16.1. The asymptotic expansion (15.4) holds true for the following cost functionals.

(1) For the functional

$$
J_{\varepsilon}(u)=\int_{\Omega} \eta(x) A \nabla\left(u-u_{d}\right) \cdot \nabla\left(u-u_{d}\right) \mathrm{d} x
$$


where $u_{0}, u_{d} \in \mathcal{C}^{6}\left([0, T] ; H^{2}(\Omega)\right) \cap \bigcap_{j=0}^{4} \mathcal{C}^{j}\left([0, T] ; H^{7-j}(\Omega)\right)$,

$$
\Lambda^{j}\left(u_{0}-u_{d}\right) \text { vanishes on } \partial \Omega \text { for } j=1,2,3,
$$

and $\eta$ is a smooth $\left(\mathcal{C}^{\infty}\right)$ function whose support does not contain $x_{0}$, we have $\delta \mathcal{J}_{1}=0$ and $\delta \mathcal{J}_{2}=0$.

(2) If we replace in (13.1) the Dirichlet boundary condition on $\partial \Omega$ by the Neumann boundary condition (for instance), then we can consider the functional

$$
\mathcal{J}_{\varepsilon}(u)=\int_{0}^{T} \eta(t) \int_{\partial \Omega}\left|u-u_{d}\right|^{2} \mathrm{~d} s \mathrm{~d} t
$$

where $u_{0 \mid \partial \Omega}, u_{d} \in \mathcal{C}^{8}\left([0, T] ; H^{-1 / 2}(\partial \Omega)\right) \cap \bigcap_{j=2}^{4} \mathcal{C}^{j}\left([0, T] ; H^{11 / 2-j}(\partial \Omega)\right)$ and $\eta$ is a smooth function whose support is contained in $[0, T)$. Then we have $\delta \mathcal{J}_{1}=0$ and $\delta \mathcal{J}_{2}=0$.

Remark 16.1. By virtue of Lemma 17.3, a sufficient condition for (16.4) to be fulfilled is

$$
\begin{gathered}
F_{0} \in \mathcal{C}^{8}\left([0, T] ; L^{2}(\Omega)\right) \cap \bigcap_{j=0}^{6} \mathcal{C}^{j}\left([0, T] ; H^{7-j}(\Omega)\right), \\
\Lambda^{j} F_{0} \text { vanishes on } \partial \Omega \text { for } j=0, \ldots, 3, \\
\Lambda^{j} u_{d} \text { vanishes on } \partial \Omega \text { for } j=0, \ldots, 3 .
\end{gathered}
$$

Remark 16.2. In the second case, due to the different nature of the boundary condition, one has slightly different regularity properties. Actually, Lemma 17.2 still holds true when an homogeneous Neumann boundary condition is applied on $\partial \Omega$, which straightforwardly leads to an analogon to Lemma 17.3. Therefore, the required regularity on $u_{0}$ is guaranteed for instance if the conditions (16.6) and (16.7) are fulfilled. Concerning the regularity of the adjoint state, i.e. to prove that (14.9) is satisfied with the assumptions made, one has to deal with a nonhomogeneous Neumann boundary condition. This is done with the help of an adaptation of Lemma 17.3 relying on a lifting of the boundary condition and a weakening of the compatibility conditions. These latter ones can be written in a form involving values of the right hand side of the PDE together with its space and time derivatives at the initial time only (the final time $T$ for the adjoint equation). They are satisfied by construction thanks to the cut-off function $\eta$.

\section{ProOfs}

\subsection{Preliminary lemmas}

We first recall two classical results. Proofs can be found in [12].

Lemma 17.1. For $0 \leq \varepsilon<\varepsilon_{0}$ ( $\varepsilon_{0}$ sufficiently small), let $Q_{\varepsilon} \in W^{1,1}\left(0, T ; H^{-1}(\Omega)\right)$ and $z_{\varepsilon}$ be the solution of

$$
\left\{\begin{aligned}
\rho_{\varepsilon} \frac{\partial^{2} z_{\varepsilon}}{\partial t^{2}}-\operatorname{div}\left(\alpha_{\varepsilon} A \nabla z_{\varepsilon}\right) & =Q_{\varepsilon} & & \text { in } \Omega \times(0, T), \\
z_{\varepsilon} & =0 & & \text { on } \partial \Omega \times(0, T), \\
z_{\varepsilon}(\cdot, 0) & =\frac{\partial z_{\varepsilon}}{\partial t}(\cdot, 0)=0 & & \text { in } \Omega .
\end{aligned}\right.
$$

There exists a constant $C>0$ such that, for all $\varepsilon \in\left[0, \varepsilon_{0}\right)$,

$$
\left\|z_{\varepsilon}\right\|_{L^{\infty}\left(0, T ; H^{1}(\Omega)\right)}+\left\|\frac{\partial z_{\varepsilon}}{\partial t}\right\|_{L^{\infty}\left(0, T ; L^{2}(\Omega)\right)} \leq C\left\|Q_{\varepsilon}\right\|_{W^{1,1}\left(0, T ; H^{-1}(\Omega)\right)}
$$


Lemma 17.2. Let $Q \in \mathcal{C}^{1}\left([0, T] ; L^{2}(\Omega)\right), z_{0} \in H^{2}(\Omega) \cap H_{0}^{1}(\Omega), z_{1} \in H_{0}^{1}(\Omega)$, and $z$ be the solution of

$$
\left\{\begin{aligned}
\rho_{0} \frac{\partial^{2} z}{\partial t^{2}}-\Lambda z & =Q & & \text { in } \Omega \times(0, T), \\
z & =0 & & \text { on } \partial \Omega \times(0, T), \\
z(\cdot, 0) & =z_{0} & & \text { in } \Omega \\
\frac{\partial z}{\partial t}(\cdot, 0) & =z_{1} & & \text { in } \Omega .
\end{aligned}\right.
$$

Then

$$
z \in \mathcal{C}\left([0, T] ; H^{2}(\Omega)\right) \cap \mathcal{C}^{1}\left([0, T] ; H_{0}^{1}(\Omega)\right) \cap \mathcal{C}^{2}\left([0, T] ; L^{2}(\Omega)\right) .
$$

This latter result can be generalized as follows.

Lemma 17.3. Let $p$ be a nonnegative integer and $Q \in \mathcal{C}^{2 p+2}\left([0, T] ; L^{2}(\Omega)\right) \cap \bigcap_{j=0}^{2 p} \mathcal{C}^{j}\left([0, T] ; H^{2 p+1-j}(\Omega)\right)$ with

$$
\Lambda^{j} Q \text { vanishing on } \partial \Omega \text { for } j=0, \ldots, p \text {. }
$$

Let $z$ be the solution of

$$
\left\{\begin{aligned}
\rho_{0} \frac{\partial^{2} z}{\partial t^{2}}-\Lambda z & =Q & & \text { in } \Omega \times(0, T), \\
z & =0 & & \text { on } \partial \Omega \times(0, T), \\
z(\cdot, 0) & =\frac{\partial z}{\partial t}(\cdot, 0)=0 & & \text { in } \Omega .
\end{aligned}\right.
$$

Then

$$
\begin{gathered}
z \in \mathcal{C}^{j}\left([0, T] ; H^{2 p+3-j}(\Omega)\right) \quad \forall j=0, \ldots, 2 p+3, \\
\Lambda^{j} z \text { vanishes on } \partial \Omega \text { for } j=0, \ldots, p .
\end{gathered}
$$

Proof. We introduce the family of auxiliary functions

$$
w_{j}=\frac{\partial^{j} z}{\partial t^{j}}, \quad j=0, \ldots, 2 p+1
$$

Using (17.3) and (17.4), it can be checked that $w_{j}$ solves:

$$
\left\{\begin{aligned}
\rho_{0} \frac{\partial^{2} w_{j}}{\partial t^{2}}-\Lambda w_{j} & =\frac{\partial^{j} Q}{\partial t^{j}} & & \text { in } \Omega \times(0, T), \\
w_{j} & =0 & & \text { on } \partial \Omega \times(0, T), \\
w_{j}(\cdot, 0) & =B_{j} & & \text { in } \Omega, \\
\frac{\partial w_{j}}{\partial t}(\cdot, 0) & =B_{j+1} & & \text { in } \Omega,
\end{aligned}\right.
$$

with

$$
\begin{aligned}
B_{2 i} & =\sum_{k=0}^{i-1} \rho_{0}^{k-i} \Lambda^{i-k-1} \frac{\partial^{2 k} Q}{\partial t^{2 k}}(0), \quad i=0, \ldots, p, \\
B_{2 i+1} & =\sum_{k=0}^{i-1} \rho_{0}^{k-i} \Lambda^{i-k-1} \frac{\partial^{2 k+1} Q}{\partial t^{2 k+1}}(0), \quad i=0, \ldots, p .
\end{aligned}
$$

Lemma 17.2 yields

$$
w_{2 p+1} \in \mathcal{C}\left([0, T] ; H^{2}(\Omega)\right) \cap \mathcal{C}^{1}\left([0, T] ; H_{0}^{1}(\Omega)\right) \cap \mathcal{C}^{2}\left([0, T] ; L^{2}(\Omega)\right),
$$

which implies by integration

$$
w_{2 p} \in \mathcal{C}^{1}\left([0, T] ; H^{2}(\Omega)\right) \cap \mathcal{C}^{2}\left([0, T] ; H_{0}^{1}(\Omega)\right) \cap \mathcal{C}^{3}\left([0, T] ; L^{2}(\Omega)\right) .
$$


Furthermore, we have

from which it follows that

$$
-\Lambda w_{2 p}=\frac{\partial^{2 p} Q}{\partial t^{2 p}}-\rho_{0} \frac{\partial^{2} w_{2 p}}{\partial t^{2}} \in \mathcal{C}\left([0, T] ; H_{0}^{1}(\Omega)\right)
$$

$$
w_{2 p} \in \mathcal{C}\left([0, T] ; H^{3}(\Omega)\right)
$$

We then obtain by bootstrapping that

$$
w_{0}=z \in \mathcal{C}^{j}\left([0, T] ; H^{2 p+3-j}(\Omega)\right) \quad \forall j=0, \ldots, 2 p+3 .
$$

By exploiting the first equation of (17.5), one can prove that

$$
\Lambda^{j} z=\rho_{0}^{j} w_{2 j}-\sum_{k=0}^{j-1} \rho_{0}^{k} \frac{\partial^{2 k}}{\partial t^{2 k}} \Lambda^{j-k-1} Q, \quad j=0, \ldots, p .
$$

Due to the hypotheses, the above function vanishes on $\partial \Omega$. This completes the proof.

Proof of Proposition 14.1. It is an application of Lemma 17.3 with $p=2$.

\subsection{Main estimate}

Let us consider the function $e_{\varepsilon}$ such that

$$
v_{\varepsilon}=v_{0}+\widehat{h}_{\varepsilon}+e_{\varepsilon},
$$

with $\widehat{h}_{\varepsilon}$ defined by (8.2).

Lemma 17.4. There holds

$$
\left\|e_{\varepsilon}\right\|_{L^{\infty}\left(0, T ; H^{1}(\Omega)\right)}=o\left(\varepsilon^{d / 2}\right) .
$$

Proof. We easily check that $e_{\varepsilon}$ solves

$$
\left\{\begin{aligned}
\rho_{1} \frac{\partial^{2} e_{\varepsilon}}{\partial t^{2}}-\alpha_{1} \operatorname{div}\left(A \nabla e_{\varepsilon}\right) & =Q_{1}+Q_{2}+Q_{3}+Q_{4} & & \text { in } \omega_{\varepsilon} \times(0, T), \\
\rho_{0} \frac{\partial^{2} e_{\varepsilon}}{\partial t^{2}}-\alpha_{0} \operatorname{div}\left(A \nabla e_{\varepsilon}\right) & =Q_{1}+Q_{4} & & \text { in }\left(\Omega \backslash \overline{\omega_{\varepsilon}}\right) \times(0, T), \\
e_{\varepsilon}^{+} & =e_{\varepsilon}^{-} & & \text {on } \partial \omega_{\varepsilon} \times(0, T), \\
\alpha_{1}\left(A \nabla e_{\varepsilon} \cdot n\right)^{+}-\alpha_{0}\left(A \nabla e_{\varepsilon} \cdot n\right)^{-} & =Q_{5} & & \text { on } \partial \omega_{\varepsilon} \times(0, T), \\
e_{\varepsilon} & =-\widehat{h}_{\varepsilon} & & \text { on } \partial \Omega \times(0, T), \\
e_{\varepsilon}(\cdot, T) & =\frac{\partial e_{\varepsilon}}{\partial t}(\cdot, T)=0 & & \text { in } \Omega,
\end{aligned}\right.
$$

where

$$
\begin{gathered}
Q_{1}=D J_{0}\left(u_{0}\right)-D J_{\varepsilon}\left(u_{0}\right), \quad Q_{2}=-\left(\rho_{1}-\rho_{0}\right) \frac{\partial^{2} v_{0}}{\partial t^{2}}, \\
Q_{3}=\left(\alpha_{1}-\alpha_{0}\right) \operatorname{div}\left(A \nabla v_{0}\right), \quad Q_{4}=-\rho_{\varepsilon} \frac{\partial^{2} \widehat{h}_{\varepsilon}}{\partial t^{2}},
\end{gathered}
$$

and for all $(x, t) \in \partial \omega_{\varepsilon} \times(0, T)$,

$$
Q_{5}(x, t)=-\left(\alpha_{1}-\alpha_{0}\right)\left(A\left[\nabla v_{0}(x, t)-\nabla v_{0}\left(x_{0}, t\right)\right] \cdot n\right) .
$$

Again we split

$$
e_{\varepsilon}=e_{1, \varepsilon}+e_{2, \varepsilon}
$$


with

$$
\left\{\begin{array}{cc}
\rho_{1} \frac{\partial^{2} e_{1, \varepsilon}}{\partial t^{2}}-\alpha_{1} \operatorname{div}\left(A \nabla e_{1, \varepsilon}\right)=Q_{1}+Q_{2}+Q_{3}+Q_{4} & \text { in } \omega_{\varepsilon} \times(0, T), \\
\rho_{0} \frac{\partial^{2} e_{1, \varepsilon}}{\partial t^{2}}-\alpha_{0} \operatorname{div}\left(A \nabla e_{1, \varepsilon}\right)=Q_{1}+Q_{4} & \text { in }\left(\Omega \backslash \overline{\omega_{\varepsilon}}\right) \times(0, T), \\
e_{1, \varepsilon}^{+}=e_{1, \varepsilon}^{-} & \text {on } \partial \omega_{\varepsilon} \times(0, T), \\
\alpha_{0}\left(A \nabla e_{1, \varepsilon} \cdot n\right)^{+}-\alpha_{1}\left(A \nabla e_{1, \varepsilon} \cdot n\right)^{-}=Q_{5} & \text { on } \partial \omega_{\varepsilon} \times(0, T), \\
e_{1, \varepsilon}=0 & \text { on } \partial \Omega \times(0, T), \\
e_{1, \varepsilon}(\cdot, T)=\frac{\partial e_{1, \varepsilon}}{\partial t}(\cdot, T)=0 & \text { in } \Omega,
\end{array}\right.
$$

and

$$
\left\{\begin{array}{cc}
\rho_{\varepsilon} \frac{\partial^{2} e_{2, \varepsilon}}{\partial t^{2}}-\operatorname{div}\left(\alpha_{\varepsilon} A \nabla e_{2, \varepsilon}\right)=0 & \text { in } \Omega \times(0, T), \\
e_{2, \varepsilon}=-\widehat{h}_{\varepsilon} & \text { on } \partial \Omega \times(0, T), \\
e_{2, \varepsilon}(\cdot, T)=\frac{\partial e_{2, \varepsilon}}{\partial t}(\cdot, T)=0 & \text { in } \Omega .
\end{array}\right.
$$

We first estimate $e_{1, \varepsilon}$. Lemma 17.1 provides

$$
\left\|e_{1, \varepsilon}\right\|_{L^{\infty}\left(0, T ; H^{1}(\Omega)\right)}+\left\|\frac{\partial e_{1, \varepsilon}}{\partial t}\right\|_{L^{\infty}\left(0, T ; L^{2}(\Omega)\right)} \leq C\left\|Q_{\varepsilon}\right\|_{W^{1,1}\left(0, T ; H^{-1}(\Omega)\right)},
$$

with

$$
Q_{\varepsilon}:=Q_{1}+Q_{4}+\left(Q_{2}+Q_{3}\right) \chi_{\omega_{\varepsilon}}+\hat{Q}_{5}
$$

and for almost all $t \in[0, T]$ and all $\varphi \in H_{0}^{1}(\Omega)$,

$$
\left\langle\hat{Q}_{5}(., t), \varphi\right\rangle_{H^{-1}(\Omega), H_{0}^{1}(\Omega)}:=\int_{\partial \omega_{\varepsilon}} Q_{5}(x, t) \varphi \mathrm{d} x .
$$

Using (13.4), (14.9) and the estimate (7.10) on $h_{\varepsilon}$, we obtain that

$$
\left\|Q_{1}+Q_{4}\right\|_{W^{1,1}\left(0, T ; H^{-1}(\Omega)\right)}=o\left(\varepsilon^{d / 2}\right) .
$$

Furthermore, it results from (14.9) together with the inequality

$$
\left\|\chi_{\omega_{\varepsilon}}\right\|_{H^{-1}(\Omega)} \leq C\left\|\chi_{\omega_{\varepsilon}}\right\|_{L^{6 / 5}(\Omega)} \leq C \varepsilon^{5 d / 6}
$$

that

$$
\left\|\left(Q_{2}+Q_{3}\right) \chi_{\omega_{\varepsilon}}\right\|_{W^{1,1}\left(0, T ; H^{-1}(\Omega)\right)}=O\left(\varepsilon^{5 d / 6}\right) .
$$

Finally, we find by similar arguments to the parabolic case that, for almost every $t \in[0, T]$,

It follows that

$$
\begin{gathered}
\left\|\hat{Q}_{5}(., t)\right\|_{H^{-1}(\Omega)} \leq C \varepsilon^{5 d / 6}\left\|v_{0}(., t)\right\|_{W^{2, \infty}(\Omega)}, \\
\left\|\frac{\partial \hat{Q}_{5}}{\partial t}(., t)\right\|_{H^{-1}(\Omega)} \leq C \varepsilon^{5 d / 6}\left\|\frac{\partial v_{0}}{\partial t}(., t)\right\|_{W^{2, \infty}(\Omega)} .
\end{gathered}
$$

Gathering (17.10)-(17.14) yields

$$
\left\|\hat{Q}_{5}\right\|_{W^{1,1}\left(0, T ; H^{-1}(\Omega)\right)} \leq C \varepsilon^{5 d / 6}\left\|v_{0}\right\|_{W^{1,1}\left(0, T ; W^{2, \infty}(\Omega)\right)} .
$$

$$
\left\|e_{1, \varepsilon}\right\|_{L^{\infty}\left(0, T ; H^{1}(\Omega)\right)}=o\left(\varepsilon^{d / 2}\right) .
$$


We now estimate $e_{2, \varepsilon}$. Let us consider again a smooth function $\theta: \Omega \rightarrow \mathbb{R}$ such that $\theta=0$ in $B\left(x_{0}, R\right)$ and $\theta=1$ on $\partial \Omega$, and set

$$
\widetilde{h}_{\varepsilon}(x, t)=\widehat{h}_{\varepsilon}(x, t) \theta(x) \quad \text { and } \quad \widetilde{e}_{2, \varepsilon}(x, t)=e_{2, \varepsilon}(x, t)+\widetilde{h}_{\varepsilon}(x, t) .
$$

The function $\widetilde{e}_{2, \varepsilon}$ solves

$$
\left\{\begin{aligned}
\rho_{\varepsilon} \frac{\partial^{2} \widetilde{e}_{2, \varepsilon}}{\partial t^{2}}-\operatorname{div}\left(\alpha_{\varepsilon} A \nabla \widetilde{e}_{2, \varepsilon}\right) & =\rho_{\varepsilon} \frac{\partial^{2} \widetilde{h}_{\varepsilon}}{\partial t^{2}}-\operatorname{div}\left(\alpha_{\varepsilon} A \nabla \widetilde{h}_{\varepsilon}\right) & & \text { in } \Omega \times(0, T), \\
\widetilde{e}_{2, \varepsilon} & =0 & & \text { on } \partial \Omega \times(0, T), \\
\widetilde{e}_{2, \varepsilon}(\cdot, T) & =\frac{\partial \widetilde{e}_{2, \varepsilon}}{\partial t}(\cdot, T)=0 & & \text { in } \Omega .
\end{aligned}\right.
$$

Lemma 17.1 provides

$$
\left\|\widetilde{e}_{2, \varepsilon}\right\|_{L^{\infty}\left(0, T ; H^{1}(\Omega)\right)} \leq C\left(\left\|\frac{\partial^{2} \widetilde{h}_{\varepsilon}}{\partial t^{2}}\right\|_{W^{1,1}\left(0, T ; H^{-1}(\Omega)\right)}+\left\|\widetilde{h}_{\varepsilon}\right\|_{W^{1,1}\left(0, T ; H^{1}(\Omega)\right)}\right) .
$$

Then, straightforward calculations lead to

$$
\left\|\tilde{e}_{2, \varepsilon}\right\|_{L^{\infty}\left(0, T ; H^{1}(\Omega)\right)} \leq C\left\|\nabla v_{0}\left(x_{0}, \cdot\right)\right\|_{W^{3,1}(0, T)}\left\|h_{\varepsilon}\right\|_{H^{1}\left(\Omega \backslash \overline{B\left(x_{0}, R\right)}\right)}=o\left(\varepsilon^{d / 2}\right) .
$$

Next,

$$
\begin{aligned}
\left\|e_{2, \varepsilon}\right\|_{L^{\infty}\left(0, T ; H^{1}(\Omega)\right)} & \leq\left\|\tilde{e}_{2, \varepsilon}\right\|_{L^{\infty}\left(0, T ; H^{1}(\Omega)\right)}+C\left\|\hat{h}_{\varepsilon}\right\|_{L^{\infty}\left(0, T ; H^{1}\left(\Omega \backslash \overline{B\left(x_{0}, R\right)}\right)\right.} \\
& \leq o\left(\varepsilon^{d / 2}\right)+C\left\|\nabla v_{0}\left(x_{0}, \cdot\right)\right\|_{L^{\infty}(0, T)}\left\|h_{\varepsilon}\right\|_{H^{1}\left(\Omega \backslash \overline{B\left(x_{0}, R\right)}\right)} \\
& \leq o\left(\varepsilon^{d / 2}\right) .
\end{aligned}
$$

This latter inequality stems from the imbedding $H^{3}(0, T) \subset L^{\infty}(0, T)$ together with Lemma 7.2. This completes the proof.

\subsection{Estimates on the direct and adjoint state}

As a consequence of Lemma 17.4, the estimates provided in Lemmas 8.2 and 8.3 remain valid in this context.

\subsection{Proof of Theorem $\mathbf{1 5 . 1}$}

We shall prove Lemmas 15.1, 15.2 and 15.3, which lead straightforwardly to the theorem. On the basis of Lemma 17.4, Lemmas 15.1 and 15.3 can be proved following the same reasoning as in the first part. Therefore we only present the proof of Lemma 15.2. We make the splitting:

$$
\int_{0}^{T}\left\langle\left(\rho_{\varepsilon}-\rho_{0}\right) \frac{\partial^{2} u_{0}}{\partial t^{2}}, v_{\varepsilon}\right\rangle_{H^{-1}(\Omega), H_{0}^{1}(\Omega)} \mathrm{d} t=\varepsilon^{d}\left(\rho_{1}-\rho_{0}\right)|\omega| \int_{0}^{T} \frac{\partial^{2} u_{0}}{\partial t^{2}}\left(x_{0}, t\right) v_{0}\left(x_{0}, t\right) \mathrm{d} t+S_{1}+S_{2}
$$

with

$$
\begin{gathered}
S_{1}=\int_{0}^{T} \int_{\omega_{\varepsilon}}\left(\rho_{1}-\rho_{0}\right) \frac{\partial^{2} u_{0}}{\partial t^{2}}\left(v_{\varepsilon}-v_{0}\right) \mathrm{d} x \mathrm{~d} t \\
S_{2}=\int_{0}^{T} \int_{\omega_{\varepsilon}}\left(\rho_{1}-\rho_{0}\right)\left[\frac{\partial^{2} u_{0}}{\partial t^{2}}(x, t) v_{0}(x, t)-\frac{\partial^{2} u_{0}}{\partial t^{2}}\left(x_{0}, t\right) v_{0}\left(x_{0}, t\right)\right] \mathrm{d} x \mathrm{~d} t .
\end{gathered}
$$


From the regularity assumptions on $u_{0}$ and $v_{0}$, it comes

$$
\left|S_{2}\right| \leq C \varepsilon^{d+1}\left\|u_{0}\right\|_{H^{2}\left(0, T ; W^{1, \infty}(\Omega)\right)}\left\|v_{0}\right\|_{L^{2}\left(0, T ; W^{1, \infty}(\Omega)\right)} .
$$

Moreover, applying the Cauchy-Schwarz inequality in time and the Hölder inequality in space together with the imbedding $H^{1}(\Omega) \subset L^{6}(\Omega)$ yields

$$
\left|S_{1}\right| \leq C \varepsilon^{5 d / 6}\left\|u_{0}\right\|_{H^{2}\left(0, T ; L^{\infty}(\Omega)\right)}\left\|v_{\varepsilon}-v_{0}\right\|_{L^{2}\left(0, T ; H^{1}(\Omega)\right)} .
$$

In view of $(8.6)$, we get that

$$
\left|S_{1}\right|=O\left(\varepsilon^{4 d / 3}\right)
$$

The proof of Lemma 15.2 is completed by gathering (17.16)-(17.18) as well as integrating by parts.

\section{REFERENCES}

[1] G. Allaire, F. de Gournay, F. Jouve and A.-M. Toader, Structural optimization using topological and shape sensitivity via a level set method. Control Cybern. 34 (2005) 59-80.

[2] H. Ammari and H. Kang, Reconstruction of small inhomogeneities from boundary measurements, Lecture Notes in Mathematics 1846. Springer-Verlag, Berlin (2004).

[3] H. Ammari and H. Kang, Reconstruction of elastic inclusions of small volume via dynamic measurements. Appl. Math. Optim. 54 (2006) 223-235.

[4] H. Ammari and H. Kang, Generalized polarization tensors, inverse conductivity problems, and dilute composite materials: a review, in Inverse problems, multi-scale analysis and effective medium theory, Contemp. Math. 408, Amer. Math. Soc., Providence, RI (2006) 1-67.

[5] S. Amstutz, Sensitivity analysis with respect to a local perturbation of the material property. Asymptotic Anal. 49 (2006) $87-108$.

[6] S. Amstutz and H. Andrä, A new algorithm for topology optimization using a level-set method. J. Comput. Phys. 216 (2006) $573-588$.

[7] S. Amstutz and N. Dominguez, Topological sensitivity analysis in the context of ultrasonic nondestructive testing. RICAM report 2005-21 (2005).

[8] S. Amstutz, I. Horchani and M. Masmoudi, Crack detection by the topological gradient method. Control Cybern. 34 (2005) 81-101.

[9] M. Bonnet, Topological sensitivity for 3d elastodynamic and acoustic inverse scattering in the time domain. Comput. Meth. Appl. Mech. Engrg. 195 (2006) 5239-5254.

[10] M. Bonnet and B.B. Guzina, Sounding of finite solid bodies by way of topological derivative. Int. J. Numer. Methods Eng. 61 (2004) 2344-2373.

[11] M. Burger, B. Hackl and W. Ring, Incorporating topological derivatives into level set methods. J. Comput. Phys. 194 (2004) 344-362.

[12] T. Cazenave and A. Haraux, Introduction aux problèmes d'évolution semi-linéaires, Mathématiques ES Applications 1. Ellipses, Paris (1990).

[13] R. Dautray and J.-L. Lions, Analyse mathématique et calcul numérique pour les sciences et les techniques, Vol. 6. INSTN: Collection Enseignement, Masson, Paris (1988).

[14] H.A. Eschenauer and A. Schumacher, Topology and shape optimization procedures using hole positioning criteria - theory and applications, in Topology optimization in structural mechanics, CISM Courses and Lectures 374, Springer, Vienna (1997) $135-196$.

[15] H.A. Eschenauer, V.V. Kobolev and A. Schumacher, Bubble method for topology and shape optimization of structures. Struct. Optimization 8 (1994) 42-51.

[16] S. Garreau, P. Guillaume and M. Masmoudi, The topological asymptotic for PDE systems: the elasticity case. SIAM J. Control Optim. 39 (2001) 1756-1778 (electronic). 
[17] P. Guillaume and K. Sid Idris, The topological asymptotic expansion for the Dirichlet problem. SIAM J. Control Optim. 41 (2002) 1042-1072 (electronic).

[18] B.B. Guzina and M. Bonnet, Topological derivative for the inverse scattering of elastic waves. Quart. J. Mech. Appl. Math. 57 (2004) 161-179.

[19] J.-L. Lions and E. Magenes, Problèmes aux limites non homogènes et applications. Vol. 2. Travaux et Recherches Mathématiques 18. Dunod, Paris (1968).

[20] S.A. Nazarov and J. Sokolowski, The topological derivative of the Dirichlet integral under the formation of a thin bridge. Siberian Math. J. 45 (2004) 341-355.

[21] G. Pólya and G. Szegö, Isoperimetric Inequalities in Mathematical Physics. Annals of Mathematics Studies 27. Princeton University Press, Princeton, N. J. (1951).

[22] J. Pommier and B. Samet, The topological asymptotic for the Helmholtz equation with Dirichlet condition on the boundary of an arbitrarily shaped hole. SIAM J. Control Optim. 43 (2004) 899-921 (electronic).

[23] M. Schiffer and G. Szegö, Virtual mass and polarization. Trans. Amer. Math. Soc. 67 (1949) 130-205.

[24] A. Schumacher, Topologieoptimierung von bauteilstrukturen unter verwendung von lopschpositionierungskriterien. Ph.D. thesis, Univ. Siegen (1995).

[25] J. Sokołowski and A. Żochowski, On the topological derivative in shape optimization. SIAM J. Control Optim. 37 (1999) 1251-1272 (electronic). 